\title{
Lithium in red giant stars: Constraining non-standard mixing with large surveys in the Gaia era ${ }^{\star, \star \star}$
}

\author{
C. Charbonnel ${ }^{1,2}$, N. Lagarde ${ }^{3}$, G. Jasniewicz ${ }^{4}$, P. L. North ${ }^{5}$, M. Shetrone ${ }^{6}$, J. Krugler Hollek ${ }^{6,9}$, V. V. Smith ${ }^{7}$, \\ R. Smiljanic ${ }^{8}$, A. Palacios ${ }^{4}$, and G. Ottoni ${ }^{1}$ \\ 1 Department of Astronomy, University of Geneva, Chemin des Maillettes 51, 1290 Versoix, Switzerland \\ e-mail: Corinne.Charbonnel@unige.ch \\ 2 IRAP, UMR 5277 CNRS and Université de Toulouse, 14, Av. E. Belin, 31400 Toulouse, France \\ 3 Institut UTINAM, CNRS UMR 6213, Univ. Bourgogne Franche-Comté, OSU THETA Franche-Comté-Bourgogne, Observatoire \\ de Besançon, BP 1615, 25010 Besançon Cedex, France \\ 4 UMR 5299 LUPM CNRS Université Montpellier II, Place Eugène Bataillon, 34095 Montpellier Cedex 05, France \\ 5 Institute of Physics, Laboratory of Astrophysics, École Polytechnique Fédérale de Lausanne (EPFL), Observatoire de Sauverny, \\ 1290 Versoix, Switzerland \\ ${ }^{6}$ University of Texas, McDonald Observatory, TX 79734, USA \\ 7 National Optical Astronomy Observatory, Tucson, USA \\ 8 Nicolaus Copernicus Astronomical Center, Polish Academy of Sciences, Bartycka 18, 00-176 Warsaw, Poland \\ 9 Twitter, 1355 Market Street, Suite 900, San Francisco, CA 94103, USA
}

Received 23 July 2019 / Accepted 22 October 2019

\begin{abstract}
Context. $\mathrm{Li}$ is extensively known to be a good tracer of non-standard mixing processes occurring in stellar interiors.

Aims. We present the results of a new large Li survey in red giant stars and combine it with surveys from the literature to probe the impact of rotation-induced mixing and thermohaline double-diffusive instability along stellar evolution.

Methods. We determined the surface Li abundance for a sample of 829 giant stars with accurate Gaia parallaxes for a large subsample (810 stars) complemented with accurate HIPPARCos parallaxes (19 stars). The spectra of our sample of northern and southern giant stars were obtained in three ground-based observatories (Observatoire de Haute-Provence, ESO-La Silla, and the Mc Donald Observatory). We determined the atmospheric parameters $\left(T_{\text {eff }}, \log (g)\right.$ and $\left.[\mathrm{Fe} / \mathrm{H}]\right)$, and the $\mathrm{Li}$ abundance. We used Gaia parallaxes and photometry to determine the luminosity of our objects and we estimated the mass and evolution status of each sample star with a maximum-likelihood technique using stellar evolution models computed with the STAREVOL code. We compared the observed Li behaviour with predictions from stellar models, including rotation and thermohaline mixing. The same approach was used for stars from selected Li surveys from the literature.

Results. Rotation-induced mixing accounts nicely for the Li behaviour in stars warmer than about $4200 \mathrm{~K}$, independently of the mass domain. For stars with masses lower than $2 M_{\odot}$ thermohaline mixing leads to further Li depletion below the $T_{\text {eff }}$ of the RGB bump (about $4000 \mathrm{~K}$ ), and on the early asymptotic giant branch, as observed. Depending on the definition we adopt, we find between 0.8 and $2.2 \%$ of Li-rich giants in our new sample.

Conclusions. Gaia puts a new spin on the understanding of mixing processes in stars, and our study confirms the importance of rotation-induced processes and of thermohaline mixing. However asteroseismology is required to definitively pinpoint the actual evolution status of Li-rich giants.
\end{abstract}

Key words. stars: abundances - stars: evolution - surveys - stars: late-type

\section{Introduction}

Along their evolution, stars modify their chemical composition through central and shell nuclear burning coupled to various internal transport mechanisms (atomic diffusion, convection, overshooting, rotation-induced mixing, thermohaline double

\footnotetext{
* Full Tables 1 and 2 are only available at the CDS via anonymous ftp to cdsarc.u-strasbg. fr (130.79.128.5) or via http: //cdsarc. u-strasbg.fr/viz-bin/cat/J/A+A/633/A34

$\star \star$ Based on observations made with the Aurélie spectrograph mounted on the $1.52 \mathrm{~m}$ telescope at Observatoire de Haute-Provence (CNRS), France, with the FEROS spectrograph mounted on the MPI $2.2 \mathrm{~m}$ telescope at ESO-La Silla Observatory (programmes 072.D0235A, B; PI: C. Charbonnel), and with the Sandiford Cassegrain Echelle Spectrometer mounted on the Struve telescope at McDonald Observatory, Texas, USA.
}

diffusion, and other kinds of hydrodynamic instabilities). These processes eventually modify the photospheric composition of the stars, in proportions that depend on their initial mass and metallicity, rotation velocity, mass loss, and evolutionary status.

In the case of low- and intermediate-mass stars (hereafter LIMS), Li-7 (hereafter Li) is one of the best tracers of the internal mixing mechanisms and of possible associated transport of angular momentum (e.g. Deliyannis et al. 2000; Talon \& Charbonnel 2010). For cool main sequence (hereafter MS) low-mass stars (LMS, i.e., masses below $\sim 1.2 \mathrm{M}_{\odot}$, which are relatively slow rotators, allowing for the precise determination of their $\mathrm{Li}$ abundance), the best constraints are the solar Li photospheric value, which is a factor of $\sim 150$ lower than the initial meteoritic value (e.g. Lodders et al. 2009; Asplund et al. 2009; Caffau et al. 2010), the Li abundance of solar-twins (e.g. King et al. 1997; 
Martín et al. 2002; Do Nascimento et al. 2009; Castro et al. 2011; Monroe et al. 2013; Carlos et al. 2016), and the so-called Li dip around effective temperature of $6700 \mathrm{~K}$ that coincides with a sharp variation in the surface rotation rate around mid-F type in open clusters like the Hyades and older (e.g. Wallerstein et al. 1965; Boesgaard \& Tripicco 1986; Boesgaard 1987; Chen et al. 2001; Cummings et al. 2017). Rotation-induced processes coupled to the effects of internal gravity waves are excellent candidates for providing a consistent explanation for the behaviour of $\mathrm{Li}$ and that of the surface and internal rotation rate of these MS G- and Ftype objects (e.g. Talon \& Charbonnel 2003, 2005; Charbonnel $\&$ Talon 2005), although additional processes like atomic diffusion, mass loss, and magnetic instabilities can play a role (e.g. Michaud 1986; Richer \& Michaud 1993; Schramm et al. 1990; Denissenkov 2010a; Eggenberger et al. 2012).

Complementary information on transport processes at play in LIMS comes from $\mathrm{Li}$ data along the subgiant and red giant branches (hereafter SGB and RGB, respectively). During these evolution phases, surface rotation is usually very low, allowing for a precise exploitation of the spectra. The low Li abundances in SGBs observed well before the occurrence of the first dredge-up (e.g. Balachandran 1995; Mallik et al. 2003; Pasquini et al. 2004; Lèbre et al. 2006; Anthony-Twarog et al. 2009) and after the end of dilution further up on the RGB (e.g. Brown et al. 1989) can be explained by rotation-induced mixing that enlarges the Li-free region inside their MS A- or F-type progenitors (e.g. Palacios et al. 2003). Later on along the RGB, the additional and sudden Li drop observed at the so-called luminosity bump (e.g. Gratton et al. 2000; Lind et al. 2009a) can be interpreted as resulting from other hydrodynamical instabilities. The most probable one is the so-called thermohaline instability, for which several alternative driving mechanisms have been proposed ("double diffusive thermohaline mode" induced by ${ }^{3} \mathrm{He}$-burning in the outer wing of the thin hydrogen-burning shell, Charbonnel \& Zahn 2007; Charbonnel \& Lagarde 2010; "magneto-thermohaline" mode driven by strong differential rotation and a poloidal magnetic field, Denissenkov et al. 2009; "cyclonic large-scale vortices" mode where rotation impacts the linear growth of the fingering instability, Sengupta \& Garaud 2018; see also phenomenological prescriptions, Henkel et al. 2017).

Several works in the literature present $\mathrm{Li}$ abundance determinations for large samples of field stars. Most of the recent studies focused on dwarf stars, looking for possible connections (which were not found) between $\mathrm{Li}$ abundance and the presence of planets (Ramírez et al. 2012, 671 FGK dwarf and subgiant stars; Delgado Mena et al. 2014, 326 dwarfs; Delgado Mena et al. 2015, 353 dwarfs). Other studies explored the case of postMS stars, starting with the pioneer work of Brown et al. (1989, 644 bright G-K giants), followed by de Laverny et al. (2003, 54 subgiant stars crossing the Hertzsprung gap), Lèbre et al. (2006, 154 bright giant stars), Luck \& Heiter (2007, 286 G/K giants), Liu et al. (2014, 378 G/K giants). Recently, large surveys have led to a paradigm shift in terms of sample size. In particular, the AMBRE and GALAH surveys have yielded public catalogues with Li abundances for, respectively, 7300 and 342000 stars covering a large range in metallicity and evolutionary status (Guiglion et al. 2016; Buder et al. 2018; Deepak 2019). A couple of studies have also searched for Li-rich stars from the Gaia-ESO survey (Casey et al. 2016, 2019; Smiljanic et al. 2018) and the LAMOST survey (Cui et al. 2012; Singh et al. 2019).

In principle, all these data can be used to study the cumulative effects of the various processes that modify the surface $\mathrm{Li}$ abundance of LIMS along their evolution. However, robust conclusions require a good knowledge of the evolution status of individual stars, and a good estimate of their mass. This was the original motivation of the present work, which aimed to derive Li abundances in a volume-limited sample of field giant stars with precise HIPPARCos parallaxes (and possibly Gaia measurements), and which are located close to or above the bump on the RGB and on the early asymptotic giant branch (AGB). Here we present the results of this observational project, and combine it with other large samples to provide robust and statistically significant constraints on stellar internal mixing processes that are suspected to be in action in LIMS along their evolution.

The paper is organised as follows. We present the selection criteria of our sample stars and determine their luminosity based on HIPPARCOS or Gaia DR2 parallaxes in Sect. 2. The observation runs and the analysis method that led to the determination of the stellar parameters and of the Li abundances are presented in Sect. 3 where we also validate our Li measurements with respect to those derived for common stars in other studies. We compare in Sect. 4 our Li data with the predictions of stellar models including rotation-induced mixing and thermohaline instability computed by some of the co-authors of the present paper (Charbonnel \& Lagarde 2010; Lagarde et al. 2012). We extend the comparison to $\mathrm{Li}$ literature data for other samples of giant stars with HIPPARCos parallaxes (Luck \& Heiter 2007; Liu et al. 2014) or Gaia parallaxes when available (GALAH survey, Buder et al. 2018). We present our conclusions in Sect. 5.

\section{Sample selection and stellar luminosities with Hipparcos and/or Gaia parallaxes}

\subsection{Initial sample selection}

Our initial goal was to use $\mathrm{Li}$ abundances in evolved stars to constrain the cumulative effects of internal mixing processes acting both on the MS and the advanced phases of the evolution of LIMS, in addition to searching for Li-rich giants. We originally selected a volume-limited sample of stars potentially located on the RGB and the early AGB, using their HIPPARCos parallaxes, $V$ magnitude, and $B-V$ and $V-I$ colours. This sample was extracted from the first HIPPARCOS catalogue (Perryman et al. 1997), requiring that the parallax be larger than 5 mas, with a relative error of less than $10 \%$. Bolometric corrections were derived from $V-I$ given in the same catalogue to estimate the luminosity of the candidates. All the stars in the sample are brighter than $V=8$. The RGB stars were selected using $V-I>0.9$ and $1.2<\log \left(L / L_{\odot}\right)<2.3$, and the possible AGB stars using $B-V<1.5$ and $\log \left(L / L_{\odot}\right)>2$.6. We favoured these two regions of the Hertzsprung-Russell diagram (HRD) because they correspond to the location of the RGB bump and that of the early-AGB and they were thought to host most of the so-called Li-rich giants (Charbonnel \& Balachandran 2000, see Sect. 4.3). To derive statistically significant features, we observed all the presumed early-AGB stars and 2/3 of the presumed RGB stars selected that way. Hence the total number of stars for which we carried out spectroscopic observations is 829 (731 probable RGBs and 98 probable AGBs).

\subsection{Determination of stellar luminosities and final sample based on Gaia DR2}

We computed the luminosity of our sample stars using parallaxes from Gaia DR2 (Gaia Collaboration 2018b) when available (810 stars out of 829), or from the New HIPPARCos catalogue 
Table 1. Informations on giants stars in our sample.

\begin{tabular}{|c|c|c|c|c|c|c|c|c|c|c|c|c|c|c|c|c|c|c|c|c|}
\hline HD & RA & Dec & $T_{\text {eff }}$ & $e\left(T_{\mathrm{eff}}\right)$ & $\mathrm{BC}$ & {$[\mathrm{Fe} / \mathrm{H}]$} & $\mathrm{A}(\mathrm{Li})$ & flag $_{\mathrm{Li}}$ & $\pi_{\mathrm{vL} 07}$ & $e\left(\pi_{\mathrm{vL} 07}\right)$ & $L_{\text {Hipp VL }}$ & $e\left(L_{\mathrm{Hipp} V \mathrm{~L}}\right)$ & $\pi_{\text {Gaia }}$ & $e\left(\pi_{\text {Gaia }}\right)$ & $\log \left(L / L_{\odot}\right)$ & $e(L)$ & flag $_{L}$ & Mass & $e_{M[-1 \sigma]}$ & $e(M[+1 \sigma])$ \\
\hline 87 & 1.4248388 & 13.396266 & 5110 & 90 & -0.23 & -0.1 & 0.8 & 0 & 8.75 & 0.3 & 1.89 & 0.03 & 8.214 & 0.1339 & 1.96 & 0.03 & 0 & 2.99 & 0.5 & 0.01 \\
\hline 645 & 2.6784778 & -12.579897 & 4980 & 130 & -0.28 & -0.1 & 0.0 & 0 & 13.33 & 0.35 & 1.43 & 0.03 & 13.881 & 0.0803 & 1.40 & 0.02 & 0 & 1.9 & 0.4 & 0.6 \\
\hline 1013 & 3.6506853 & 20.206701 & 3600 & 150 & -2.24 & 0.0 & -1.1 & -1 & 8.86 & 0.22 & 2.98 & 0.02 & 9.1895 & 0.4342 & 3.01 & 0.06 & 0 & 1.52 & 0.71 & 1.46 \\
\hline 1483 & 4.6773424 & -43.23557 & 4420 & 110 & -0.58 & -0.1 & 0.0 & 0 & 8.89 & 0.49 & 1.70 & 0.05 & 7.3177 & 0.0392 & 1.88 & 0.02 & 0 & 1.18 & 0.31 & 0.67 \\
\hline 2261 & 6.571046 & -42.30598 & 4770 & 250 & -0.37 & -0.4 & 0.2 & 0 & 38.5 & 0.73 & 1.92 & 0.02 & -9999.0 & -9999.0 & 1.93 & 0.02 & 1 & 1.57 & 0.49 & 1.16 \\
\hline 2529 & 7.179697 & -50.53286 & 4650 & 110 & -0.43 & -0.1 & 0.3 & 0 & 7.98 & 0.43 & 1.76 & 0.05 & 8.4914 & 0.0322 & 1.72 & 0.02 & 0 & 1.41 & 0.46 & 1.08 \\
\hline 3712 & 10.126836 & 56.53733 & 4510 & 90 & -0.51 & -0.4 & 0.0 & 0 & 14.29 & 0.15 & 2.89 & 0.01 & 1.6949 & 1.2847 & 4.38 & 0.68 & 0 & 5.92 & 0.0 & 0.01 \\
\hline 3750 & 9.966457 & -44.79629 & 4610 & 110 & -0.45 & 0.0 & 2.5 & 0 & 10.62 & 0.43 & 1.63 & 0.04 & 10.2314 & 0.0489 & 1.67 & 0.02 & 0 & 1.49 & 0.5 & 1.0 \\
\hline 4042 & 11.012434 & 70.823425 & 5020 & 120 & -0.26 & -0.3 & 1.1 & 0 & 6.8 & 0.32 & 1.64 & 0.04 & 6.9952 & 0.0288 & 1.62 & 0.02 & 0 & 2.0 & 0.9 & 0.5 \\
\hline 4211 & 11.050414 & -38.421684 & 4600 & 100 & -0.46 & 0.0 & 0.0 & 0 & 9.63 & 0.4 & 1.76 & 0.04 & 10.1675 & 0.0691 & 1.72 & 0.02 & 0 & 1.54 & 0.5 & 0.96 \\
\hline 4229 & 9.914577 & -85.700874 & 4340 & 90 & -0.64 & 0.0 & 0.0 & -1 & 6.94 & 0.32 & 1.75 & 0.04 & 7.0051 & 0.0235 & 1.75 & 0.023 & 0 & 1.09 & 0.26 & 0.71 \\
\hline 4496 & 11.605771 & -57.927643 & 4510 & 90 & -0.51 & 0.0 & 0.0 & 0 & 6.71 & 0.57 & 1.56 & 0.08 & 4.912 & 0.0522 & 1.83 & 0.03 & 0 & 1.44 & 0.43 & 1.05 \\
\hline 4585 & 11.930084 & -18.061338 & 4290 & 110 & -0.7 & -0.2 & -0.5 & -1 & 9.18 & 0.41 & 1.97 & 0.04 & 8.8769 & 0.1143 & 2.03 & 0.03 & 0 & 1.02 & 0.21 & 0.64 \\
\hline 5316 & 13.811135 & 24.557062 & 3400 & 150 & -3.2 & -0.4 & -1.3 & -1 & 5.75 & 0.42 & 3.18 & 0.07 & 4.5201 & 0.1855 & 3.40 & 0.05 & 0 & 1.31 & 0.6 & 1.66 \\
\hline 5395 & 14.166271 & 59.181057 & 5030 & 260 & -0.26 & -0.4 & 0.0 & 0 & 16.32 & 0.23 & 1.73 & 0.02 & 17.2875 & 0.188 & 1.72 & 0.03 & 0 & 1.9 & 0.89 & 0.6 \\
\hline 5457 & 13.751303 & -69.527084 & 4680 & 90 & -0.42 & 0.0 & 0.0 & 0 & 14.7 & 0.27 & 1.55 & 0.02 & 14.6214 & 0.0817 & 1.58 & 0.02 & 0 & 1.59 & 0.65 & 0.9 \\
\hline 5722 & 14.682782 & -11.379975 & 5100 & 120 & -0.24 & -0.2 & 0.0 & -1 & 10.2 & 0.53 & 1.73 & 0.05 & 9.7623 & 0.112 & 1.79 & 0.028 & 0 & 2.49 & 0.56 & 0.5 \\
\hline 6559 & 16.532099 & -23.99245 & 4730 & 90 & -0.39 & -0.1 & 0.0 & 0 & 8.52 & 0.44 & 1.75 & 0.05 & 8.8661 & 0.0578 & 1.72 & 0.02 & 0 & 1.59 & 0.75 & 0.9 \\
\hline
\end{tabular}

Notes. flag $\mathrm{L}_{\mathrm{Li}}$ indicates the determination and the upper limit of Li abundances $\left(0\right.$ and -1 , respectively). flag $\mathrm{g}_{L}$ indicates the method used to compute the luminosity $\log \left(L / L_{\odot}\right)\left(0: \pi_{\mathrm{DR} 2}+V_{\mathrm{Evans} 18} ; 1: \pi_{\mathrm{v} 107}+V_{\mathrm{Tycho}} ; 2: \pi_{\mathrm{v} 107}+V_{\mathrm{Hipp} 97} ; 0: \pi_{\mathrm{DR} 2}+V_{\mathrm{Tycho}}\right) . e_{M[-1 \sigma]}$ and $e_{M[+1 \sigma]}$ correspond to the error on the mass determination at $-1 \sigma$ and $+1 \sigma$, respectively. The full table is available at the CDS.

(van Leeuwen 2007) for the remainder (19 stars). We adopted $V$ magnitude from Evans et al. (2018) when available (800 stars), from Tycho-2 (25 stars) or van Leeuwen (2007, 4 stars) catalogues otherwise. In all cases we applied the bolometric correction relation of Flower (1996). We provide the corresponding luminosity value for each individual star in Table 1, together with flags indicating the origin of the parallax and of the $V$ magnitude $\left(\right.$ flag $\left._{L}\right)$. We adopted these values of the luminosity for our analysis and use them to plot the positions of our sample stars in the HRD (Figs. 1, 4, 7, 8 and 18). In Fig. 1 we differentiate the stars that have Gaia DR2 parallaxes better than $10 \%$ (805 out of 810; 5 stars have errors between 10 and $20 \%$, and 2 outliers have errors larger than 70\%); the 19 stars with no Gaia DR2 parallaxes have New HIPPARCos parallaxes better than $10 \%$. The apparent lack of stars with $\log \left(L / L_{\odot}\right)$ between $\sim 2.3$ and 2.6 results from our sample selection.

For a consistency check, we also computed the luminosity using the parallaxes and $V$ magnitudes from van Leeuwen (2007) for the entire sample, assuming a bolometric correction relation of Flower (1996). The corresponding luminosity values are also given in Table 1 and compared to the previous ones in Fig. 2. Except in a few cases, the agreement between the two sets of luminosity is reasonable (e.g. within \pm 0.2 dex). We refer to the original Gaia DR2 papers (Gaia Collaboration 2018a,b; Lindegren et al. 2018) for general discussions on the differences between their catalogue and that of van Leeuwen (2007).

\section{Observations and spectra processing}

\subsection{Observations and data reduction}

Several observing runs were performed to secure the original data set built for the present study with the selection criteria described in Sect. 2.

Three observing runs were performed at the Observatoire de Haute Provence, from 8 September to 15 September 2003 (visitor mode), from 17 January to 19 January, 2004 (service mode), and from 16 January to 16 February, 2004, service mode). High-resolution spectra were obtained with the Aurélie spectrograph (Gillet et al. 1994) attached to the $1.52 \mathrm{~m}$ telescope. Since Aurélie has no cross-disperser, we selected the spectral range centred on the $\mathrm{Li} \lambda 670.8 \mathrm{~nm}$ line and about $20 \mathrm{~nm}$ wide.
The Aurélie spectrograph uses a cooled 2048-photodiode detector forming a $13 \mu \mathrm{m}$ pixel linear array. The entrance spherical diaphragm is $3^{\prime \prime}$ wide. For the spectral range centred on $\lambda 670.8 \mathrm{~nm}$, the grating \#5 with 1200 grooves $\mathrm{mm}^{-1}$ was used, giving a mean dispersion of $0.26 \mathrm{~nm} \mathrm{~mm}^{-1}$ at order 2 , a resolution $R=70000$ and a spectral coverage of about $7 \mathrm{~nm}$. The order-separating filter was the OG515. At the beginning, end, and middle of each night, thorium lamps were observed for wavelength calibration, while at the beginning and end of each night, a series of nine flat-fields were obtained using an internal lamp (Tungsten), as well as a series of five offsets. These spectra were reduced using standard MIDAS procedures. The exposure times were adjusted to obtain a signal-to-noise ratio $(\mathrm{S} / \mathrm{N})$ higher than $\sim 150$ per pixel of the extracted spectrum in the $\mathrm{Li}$ line region.

Two observing runs were performed in visitor mode at ESOLa Silla, Chile, from 3 January to 6 January, 2004, and from 5 March to 8 March, 2004. High resolution $(R \simeq 43000)$ echelle spectra were obtained with the FEROS spectrograph attached to the MPG/ESO $2.2 \mathrm{~m}$ telescope. Each spectrum covers the whole visible range and even beyond, between about 400 and $900 \mathrm{~nm}$. All spectra were reduced using the ESO pipeline FEROS-DRS which runs within MIDAS. The typical $\mathrm{S} / \mathrm{N}$ obtained is $150-200$ per pixel of the extracted spectrum in the Li region.

A total of 92 stars were observed at the McDonald Observatory with the Otto Struve Telescope using the Sandiford Cassegrain Echelle Spectrometer (SES) from August 2003 to May 2004 over the course of seven observing runs. The \#9 slit $\left(1.1^{\prime \prime} \times 3.5^{\prime \prime}\right)$ was used to yield a two pixel resolution of 60000 . After each star's observation, a ThAr exposure and a flat field were taken for wavelength calibration purposes. The wavelength region spans from 618 to $815 \mathrm{~nm}$ in the red portion of the spectra. The data were reduced using the standard echelle routines in IRAF.

\subsection{Determination of effective temperature and gravity}

All spectra coming from the OHP, ESO, and MacDonald observatories were processed in the same way. Since spectra analysis was done well before Gaia DR2, we took the required quantities listed below from the HIPPARCos catalogue. 


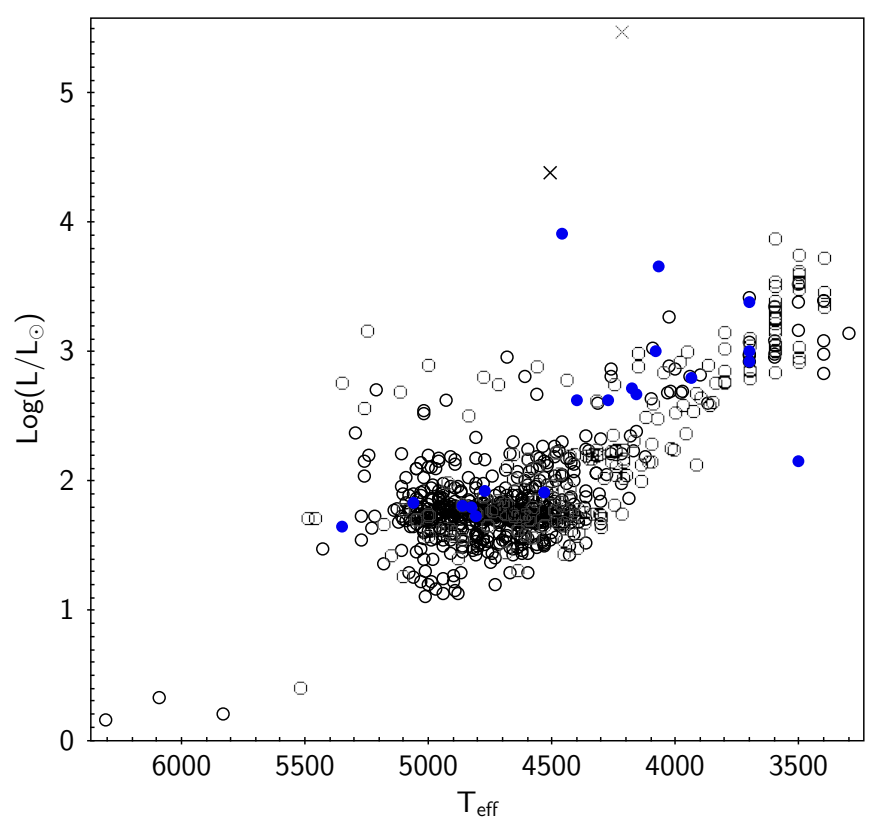

Fig. 1. Positions in HRD of our entire sample. Stars with Gaia DR2 parallaxes are shown by black open symbols (circles and crosses for parallaxes better or worse than than $10 \%$, respectively); blue symbols are stars with New HIPPARcos parallaxes only (all better than $10 \%$ ). Input parameters from the different catalogues used for the determination of the stellar luminosities and effective temperatures are described in Sects. 2.2 and 3.2 respectively.

For each star, the effective temperature $T_{\text {eff }}$ was determined by using both $B-V$ and $V-I$ colours from the New HIPPARCos catalogue (van Leeuwen 2007) and the colour-temperature relations for F-K giants from Houdashelt et al. (2000). For the coolest stars $\left(T_{\text {eff }} \leq 4000 \mathrm{~K}\right)$ we used Alonso et al. (1999). Reddening was low for typical distances of the order of $100 \mathrm{pc}$ and can be neglected as done by Luck \& Heiter (2007). The error on $T_{\text {eff }}$ was estimated from an interpolation processing in the Houdashelt tabular data assuming $2.0 \leq \log g \leq 3.0$. The stellar effective temperatures and the corresponding errors are given in Table 1 and they are used across different figures throughout the paper. We compare our values for the effective temperature with those of Gaia DR2 (Gaia Collaboration 2018b) in Fig. 3. As expected, the largest differences are found among the coolest stars with effective temperature below $\sim 4000 \mathrm{~K}$ (see e.g. Andrae et al. 2018; Arenou et al. 2018). The cut at $4000 \mathrm{~K}$ mentioned above in our $T_{\text {eff }}$ calibration procedure also reinforces the observed discrepancies in Fig. 3.

For the spectra analysis we consider bolometric magnitude $M_{\text {bol }}$ and gravity $\log (g)$ as given by $M_{\text {bol }}=V-3.1 E(B-V)+$ $B C(V)+5 \log (\pi)+5$ and $\log (g)=\log (M)+0.4 M_{\text {bol }}+4 \log \left(T_{\text {eff }}\right)-$ 12.505 using the 1997 HIPPARCos parallax $\pi$ and $M=2 M_{\odot}$ for all G-K giant stars; the error on $\log (g)$ comes essentially from the error on mass.

\subsection{Determination of $[\mathrm{Fe} / \mathrm{H}]$ and of $\mathrm{Li}$ abundances}

All spectra and calibration files were processed with the MIDAS package. For each star, a series of synthetic spectra with effective temperature $\log \left(T_{\text {eff }}\right)$ and gravity $\log (g)$ derived as described in Sect. 3.2 and with various metallicities $(-0.6 \leqq[\mathrm{Fe} / \mathrm{H}] \leqq 0.2)$ were calculated between 665 and $675 \mathrm{~nm}$ with tools from the Uppsala Stellar Atmospheres Group (Gustafsson et al. 2008). The MARCS model atmospheres used here are line-blanketed

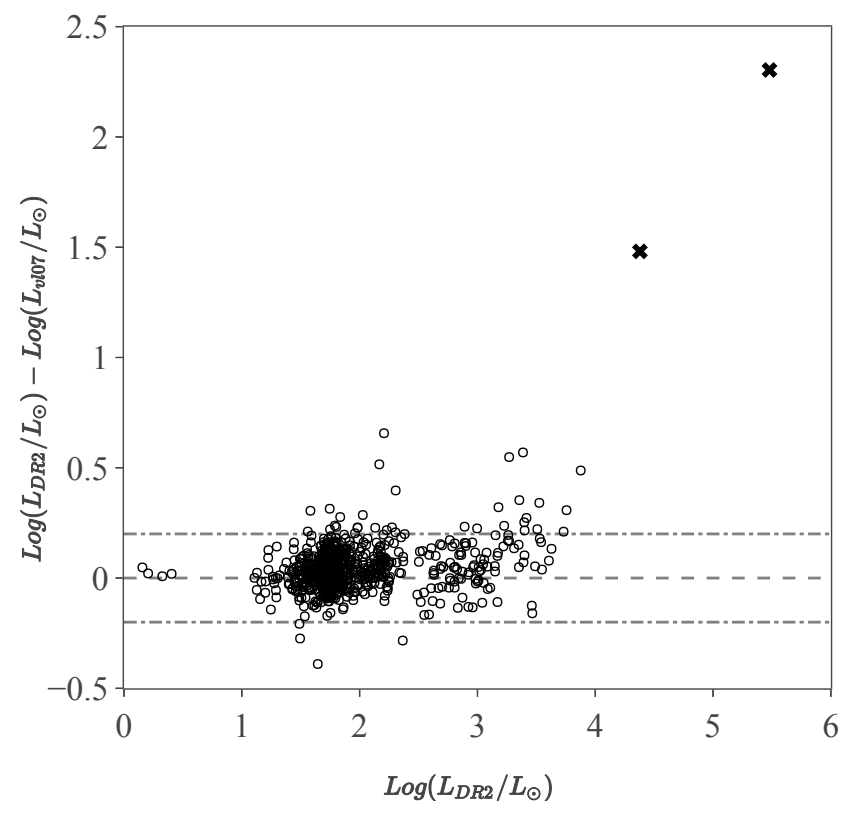

Fig. 2. Comparison between two sets of luminosity values derived for our sample stars using Gaia DR2 or New HIPPARCos parallaxes as described in Sect. 2.2. Circles and crosses correspond to stars with Gaia DR2 parallaxes better or worse than than $10 \%$, respectively. The dashed-dotted lines represent a difference of 0.2 dex.

local thermodynamic equilibrium (LTE) models that include spherical symmetry, the opacity sampling treatment of line opacities, a chemical equilibrium extended to a few hundred molecular species, opacity for $\mathrm{TiO}$ and $\mathrm{H}_{2} \mathrm{O}$, and updates of the continuous and atomic opacities (Gustafsson et al. 2008). We adopted a uniform micro-turbulent velocity of $2.0 \mathrm{~km} \mathrm{~s}^{-1}$ for all synthetic spectra. The model spectra use the line list around $671 \mathrm{~nm}$ extensively described by Lèbre et al. (1999). With the help of the computed synthetic spectra, some short spectral windows where the continuum approaches unity were identified in our observed spectra and used to draw the continuum. After the normalisation of observed spectra by their continuum, corrections for radial velocities were performed by using a MIDAS cross-correlation procedure between observed and synthetic spectra. Mean metallicity and Li abundance are determined for each star by the best fit between the normalised spectrum and synthetic spectra: $[\mathrm{Fe} / \mathrm{H}]$ was at first derived from metallic lines (mostly from about thirty Fe lines between 665 and $675 \mathrm{~nm}$ ), and then $\mathrm{A}(\mathrm{Li})^{1}$ is derived from the resonance LiI $\lambda 670.8 \mathrm{~nm}$ line. The error on the mean metallicity estimated from the fit of the iron lines found in the vicinity of the Li line is around 0.1 dex. The final adopted uncertainty of the $\mathrm{Li}$ abundance is around 0.2 dex.

Abundances of $\mathrm{Li}$ and $[\mathrm{Fe} / \mathrm{H}]$ values are given in Table 1 . An indication of the $\mathrm{Li}$ range for all individual stars is presented in the HRD in Fig. 4. [Fe/H] values are shown as a function of effective temperature in Fig. 5. Respectively 79.2, 20.6 , and $0.2 \%$ of our sample stars have $-0.2 \leq[\mathrm{Fe} / \mathrm{H}] \leq+0.2$, $[\mathrm{Fe} / \mathrm{H}]<-0.2$, and $[\mathrm{Fe} / \mathrm{H}]>+0.2 \quad(29 \%$ of the total have $[\mathrm{Fe} / \mathrm{H}]=0)$.

The Li abundances given here are computed in LTE. We are aware that non-LTE (NLTE) effects are not negligible, especially on the Li I $\lambda 6708$ line used here for abundance determination. Lind et al. (2009b) have computed NLTE corrections for giants

$1 \mathrm{~A}(\mathrm{Li})=\log \left(\frac{\mathrm{X}(\mathrm{Li})}{\mathrm{X}(\mathrm{H})} \cdot \frac{A_{\mathrm{H}}}{A_{\mathrm{Li}}}\right)+12$, with $\mathrm{X}(\mathrm{Li})$ the lithium mass fraction. 


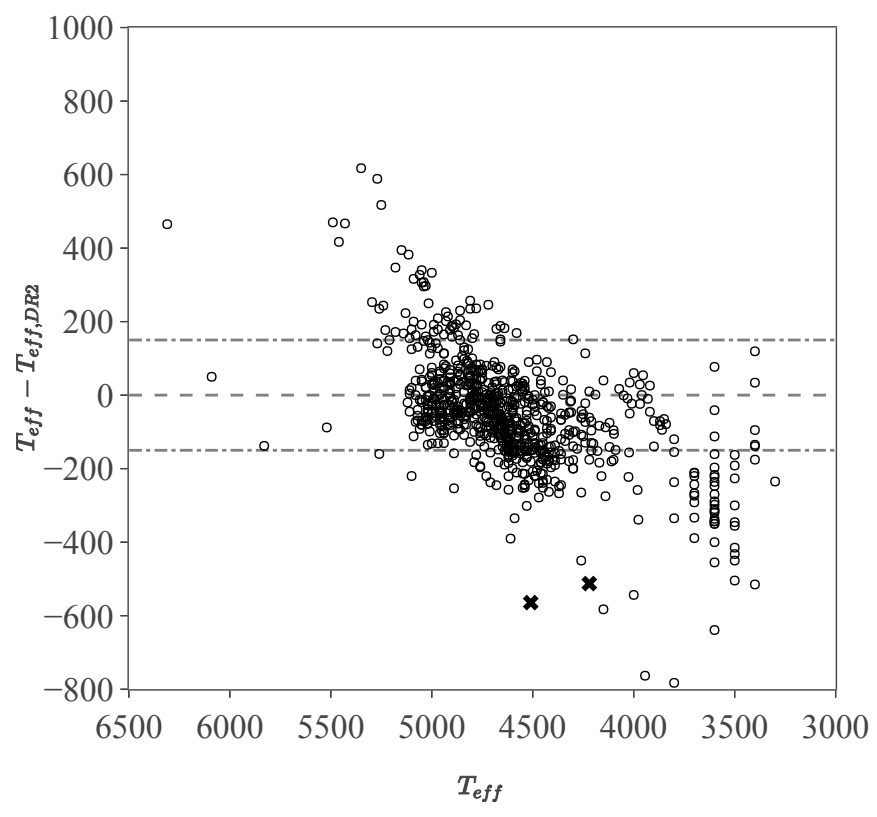

Fig. 3. Comparison between effective temperature determined here and that published in Gaia DR2 for our sample stars (same symbols as in Fig. 2). Dashed-dotted lines represent a difference of $150 \mathrm{~K}$ in $T_{\text {eff }}$.

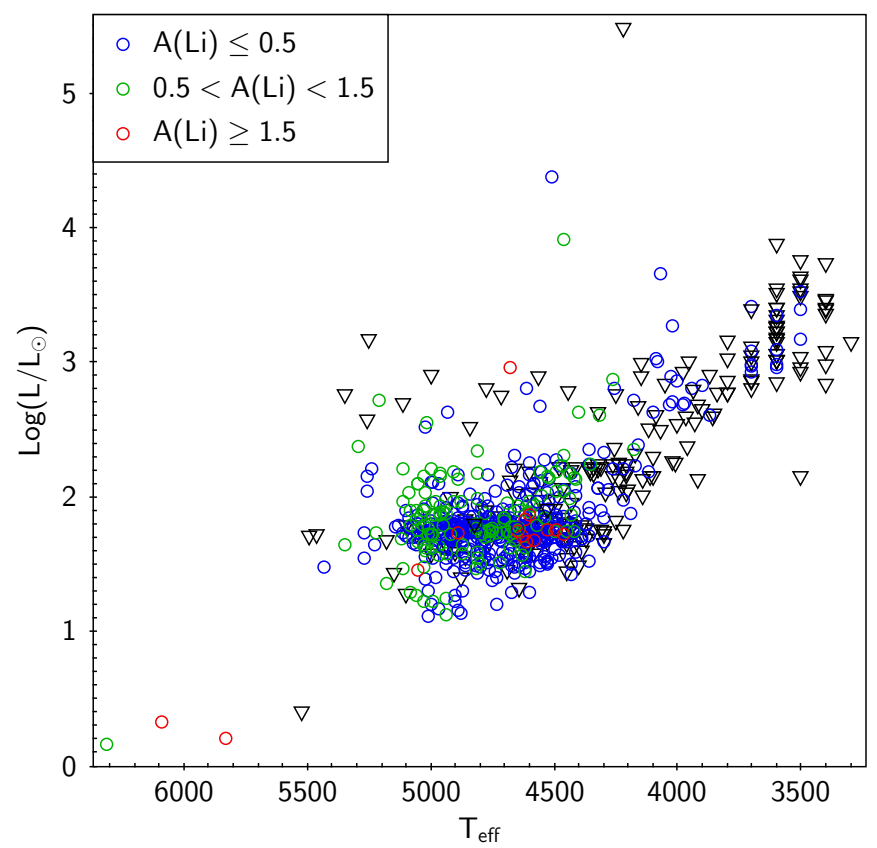

Fig. 4. Indication of range in Li content (LTE; colour-labelled; circles are for abundance determinations and triangles are for upper limits) derived in this work for all individual stars of our sample with their positions in HRD. Luminosities are computed as in Fig. 1 (see text for more).

with $T_{\text {eff }}$ between $4000 \mathrm{~K}$ and $8000 \mathrm{~K}$ and various metallicities, surface gravities, and $\mathrm{Li}$ abundances. We did not apply these corrections to our LTE abundances, particularly as we would have had to extrapolate them for stars with $T_{\text {eff }}<4000 \mathrm{~K}$, that is, for about $10 \%$ of our sample. Furthermore, the grid covers $\mathrm{Li}$ abundances down to $\mathrm{A}(\mathrm{Li})=-0.3$, while our observed abundances or upper limits are lower than this value for several tens of stars. As shown by Liu et al. (2014), the NLTE correction $\Delta_{\mathrm{NLTE}} \equiv A_{\mathrm{NLTE}}-A_{\mathrm{LTE}}$ depends above all on $T_{\mathrm{eff}}:$ while $\Delta_{\mathrm{NLTE}}$ amounts to only about $0.1 \mathrm{dex}$ for $T_{\text {eff }} \geq 5200 \mathrm{~K}$, it reaches

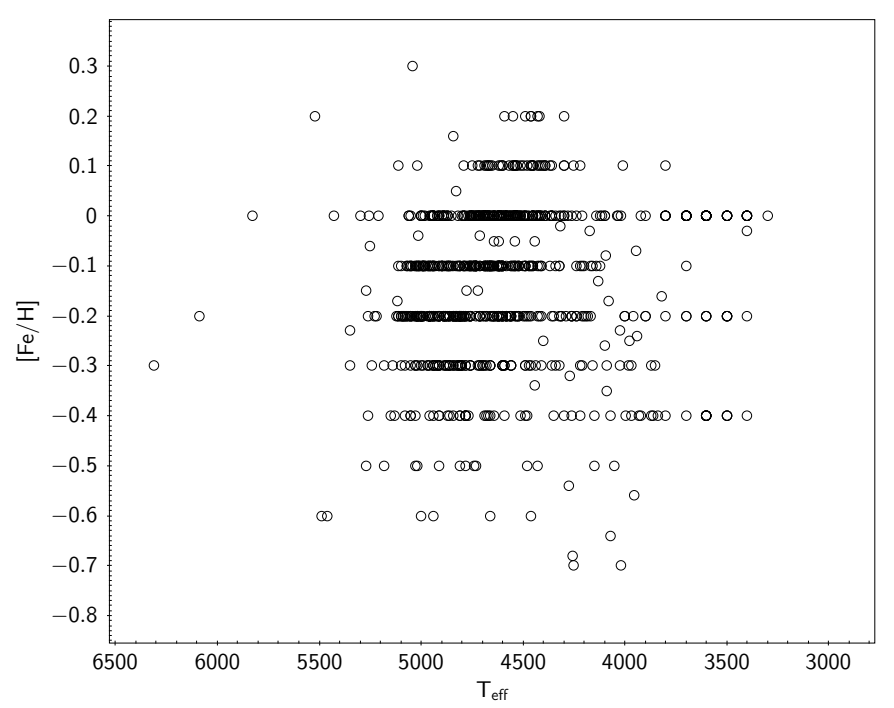

Fig. 5. $[\mathrm{Fe} / \mathrm{H}]$ as function of effective temperature for our sample stars.

about $0.3 \mathrm{dex}$ at $T_{\text {eff }}=4500 \mathrm{~K}$. For cooler stars, which represent roughly $1 / 3$ of our sample, Table 2 of Lind et al. (2009b) displays NLTE corrections as large as 0.33 dex for solar metallicity stars with $T_{\text {eff }}=4000 \mathrm{~K}, \log g=2.0,[\mathrm{Fe} / \mathrm{H}]=0.0$ and $\mathrm{A}(\mathrm{Li})=0.0$ (for a $2 \mathrm{~km} \mathrm{~s}^{-1}$ microturbulence). For higher effective temperatures, the corrections are smaller but always positive as long as $\mathrm{A}(\mathrm{Li}) \leq 2.7$. In other words, our LTE Li abundances underestimate the true abundances by a few tenths of a dex at most. We will comment this briefly in Sect. 4 .

\subsection{Comparisons with other studies}

We have two, eight, and eighty two stars, respectively, in common with the samples of de Laverny et al. (2003), Lèbre et al. (2006), and Luck \& Heiter (2007). We have nine stars in common with the Li sample of the AMBRE project (Guiglion et al. 2016); however the stellar effective temperatures are not provided in this study, and only two out of the nine common stars have had their $[\mathrm{Fe} / \mathrm{H}]$ values published after being determined with AMBRE tools (Mikolaitis et al. 2017). We have no stars in common with the samples of Brown et al. (1989), Liu et al. (2014), nor the large GALAH survey (Buder et al. 2018).

We show in Fig. 6 the comparison between our LTE Li results and the LTE $\mathrm{Li}$ values of the above-mentioned papers which include the same stars (see caption for details). We also compare the effective temperature values (since the Li abundance is more sensitive to this parameter than to others, and because $T_{\text {eff }}$ is important in the comparison with stellar models, see Sect. 4.2) and the $[\mathrm{Fe} / \mathrm{H}]$ values.

Our Li and $T_{\text {eff }}$ determinations agree fairly well (within less than 0.2 dex and $150 \mathrm{~K}$ respectively) with those derived by Luck \& Heiter (2007) through their photometric pipeline. This was expected since we used the same photometric processing (calibration using observed $B-V$ and Houdashelt et al. 2000 models for the determination effective temperature) and the same MARCS models for determining the Li abundances. Larger differences exist, however, between our analysis and the works of Lèbre et al. (2006) and de Laverny et al. (2003). These two studies relied on stellar parameters from the literature, which might have introduced some heterogeneity in their analysis; additionally, Lèbre et al. (2006) report smaller $\mathrm{S} / \mathrm{N}$ than we do here ( $>50$ for northern stars, $>80$ for southern stars), and their Aurélie 
Table 2. Informations on giants stars in three other samples used in this study.

\begin{tabular}{|c|c|c|c|c|c|c|c|c|}
\hline Name & $T_{\text {eff }}$ & $\pi_{\text {Gaia }}$ & $e\left(\pi_{\text {Gaia }}\right)$ & $\log \left(L / L_{\odot}\right)$ & $e(L)$ & Mass & $e(M[-1 \sigma])$ & $e(M[+1 \sigma])$ \\
\hline \multicolumn{9}{|c|}{ Luck \& Heiter (2007) } \\
\hline 448 & 4840 & 10.9923 & 0.1299 & 1.74 & 0.03 & 2.49 & 1.1 & 0.5 \\
\hline 3411 & 4657 & 8.7765 & 0.0385 & 1.71 & 0.02 & 2.49 & 1.28 & 0.5 \\
\hline 3546 & 5102 & 19.4949 & 0.2307 & 1.71 & 0.03 & 2.0 & 1.09 & 0.5 \\
\hline 4732 & 5021 & 18.2248 & 0.0565 & 1.13 & 0.02 & 1.9 & 0.3 & 0.1 \\
\hline 5722 & 4995 & 9.7623 & 0.112 & 1.80 & 0.03 & 2.49 & 0.84 & 0.5 \\
\hline 6037 & 4669 & 10.688 & 0.044 & 1.42 & 0.02 & 1.59 & 0.6 & 0.9 \\
\hline 6186 & 4955 & 17.2961 & 0.3842 & 1.87 & 0.04 & 2.49 & 0.95 & 0.5 \\
\hline 6559 & 4773 & 8.8661 & 0.0578 & 1.70 & 0.02 & 2.0 & 1.15 & 0.99 \\
\hline 7106 & 4748 & 19.2161 & 0.2278 & 1.71 & 0.03 & 1.9 & 0.9 & 1.09 \\
\hline 7578 & 4715 & 9.3814 & 0.0538 & 1.70 & 0.02 & 2.49 & 1.4 & 0.5 \\
\hline 8512 & 4771 & 28.6441 & 0.413 & 1.72 & 0.03 & 1.79 & 0.94 & 1.19 \\
\hline$\ldots$ & $\ldots$ & $\ldots$ & $\ldots$ & $\ldots$ & $\ldots$ & $\ldots$ & $\ldots$ & $\ldots$ \\
\hline \multicolumn{9}{|c|}{ Liu et al. (2014) } \\
\hline 87 & 5072 & 8.214 & 0.1339 & 1.97 & 0.03 & 2.99 & 0.5 & 0.01 \\
\hline 360 & 4850 & 8.9376 & 0.0836 & 1.74 & 0.03 & 2.48 & 1.5 & 0.51 \\
\hline 448 & 4780 & 10.9923 & 0.1299 & 1.75 & 0.03 & 2.48 & 1.46 & 0.51 \\
\hline 587 & 4893 & 17.3901 & 0.1339 & 1.21 & 0.02 & 1.7 & 0.5 & 0.3 \\
\hline 645 & 4880 & 13.881 & 0.0803 & 1.41 & 0.02 & 2.0 & 0.5 & 0.5 \\
\hline 1239 & 5114 & 4.7303 & 0.0955 & 2.36 & 0.03 & 3.98 & 1.0 & 0.02 \\
\hline 1367 & 4982 & 8.875 & 0.0419 & 1.65 & 0.02 & 2.49 & 0.7 & 0.5 \\
\hline 1419 & 4888 & 8.5872 & 0.1468 & 1.73 & 0.03 & 2.49 & 0.6 & 0.5 \\
\hline 2114 & 5230 & 5.4912 & 0.0949 & 2.20 & 0.03 & 2.99 & 0.01 & 1.0 \\
\hline 2952 & 4844 & 9.054 & 0.0535 & 1.75 & 0.02 & 2.49 & 1.39 & 0.5 \\
\hline 3421 & 5287 & 3.5023 & 0.1105 & 2.73 & 0.04 & 3.97 & 0.01 & 0.99 \\
\hline$\ldots$ & $\ldots$ & $\ldots$ & $\ldots$ & $\ldots$ & $\ldots$ & $\ldots$ & $\ldots$ & $\ldots$ \\
\hline \multicolumn{9}{|c|}{ GALAH Buder et al. (2018) } \\
\hline 4634991546064263040 & 4064 & 0.255 & 0.0193 & 2.55 & 0.08 & 1.24 & 0.44 & 1.24 \\
\hline 4644232799560251776 & 4052 & 0.2225 & 0.0243 & 2.43 & 0.11 & 1.0 & 0.23 & 0.87 \\
\hline 4642633461114384640 & 4176 & 0.1932 & 0.0271 & 2.29 & 0.14 & 0.92 & 0.15 & 0.93 \\
\hline 4622338434971333248 & 4388 & 0.3384 & 0.0196 & 1.93 & 0.07 & 0.98 & 0.2 & 0.55 \\
\hline 4622323316686362752 & 4065 & 0.2681 & 0.0262 & 2.57 & 0.10 & 1.46 & 0.63 & 1.26 \\
\hline 2956458403404947712 & 4461 & 0.1282 & 0.0167 & 2.36 & 0.13 & 1.11 & 0.34 & 1.36 \\
\hline 4827471673666050816 & 4188 & 0.2602 & 0.0192 & 2.26 & 0.08 & 1.02 & 0.21 & 0.83 \\
\hline 4800298412016784896 & 4291 & 0.2295 & 0.0208 & 2.19 & 0.10 & 1.65 & 0.76 & 1.34 \\
\hline 2890175650594498048 & 4050 & 0.1305 & 0.0227 & 2.99 & 0.17 & 2.47 & 1.55 & 1.49 \\
\hline 2890162456454924544 & 4434 & 0.1656 & 0.0271 & 2.45 & 0.16 & 2.99 & 1.29 & 1.98 \\
\hline 2893967522602401792 & 4391 & 0.2155 & 0.0234 & 2.24 & 0.11 & 2.48 & 1.38 & 1.5 \\
\hline$\ldots$ & $\ldots$ & $\ldots$ & $\ldots$ & $\ldots$ & $\ldots$ & $\ldots$ & $\ldots$ & $\ldots$ \\
\hline
\end{tabular}

Notes. We use HD number to identify stars observed by Luck \& Heiter (2007) and Liu et al. (2014) while the Gaia identify is used for the GALAH sample. The full table is available at the CDS.

spectra for northern stars have a lower resolution (45 000 instead of 70000$)$, but their CES@CAT spectra have a higher resolution than ours (95000). The study of de Laverny et al. (2003) relies on the same observational material.

The bottom panel of Fig. 6 shows that our estimates of $[\mathrm{Fe} / \mathrm{H}]$ tend to be lower than those of other authors by about 0.1 to 0.3 dex. The explanation for that difference certainly lies in the $2.0 \mathrm{~km} \mathrm{~s}^{-1}$ micro-turbulence value we adopted, and which is larger by about $0.3 \mathrm{~km} \mathrm{~s}^{-1}$ on average than the typical values determined by Luck \& Heiter (2007). Indeed, a larger micro-turbulent velocity results in a lower abundance, since the resulting synthetic lines are less saturated. The other two works involved in Fig. 6 adopted $v_{\text {turb }}=2.0 \mathrm{~km} \mathrm{~s}^{-1}$ but their contribution amounts to only 10 stars instead of 82 for Luck \& Heiter (2007).

\section{Comparison with stellar model predictions}

\subsection{Mass range and evolution status of the sample stars}

In order to test model predictions for the Li behaviour along stellar evolution with respect to observations, we need to estimate the mass of individual stars. For this, we applied the following method to our sample and to the samples of Luck \& Heiter (2007), Liu et al. (2014), and Buder et al. (2018). We use a maximum-likelihood technique adapted from Valle et al. (2014) that compares the effective temperature, luminosity, and $[\mathrm{Fe} / \mathrm{H}]$ of individual stars to theoretical evolution tracks in the HRD. The interpolation is done between the standard models (without rotation) of Lagarde et al. $(2017,2019)$ that are available for six values of $[\mathrm{Fe} / \mathrm{H}](0.51,0,-0.23,-0.54,-1.2,-2.14)$ for a range of masses between 0.6 and $6 \mathrm{M}_{\odot}$. The mass estimate accounts 

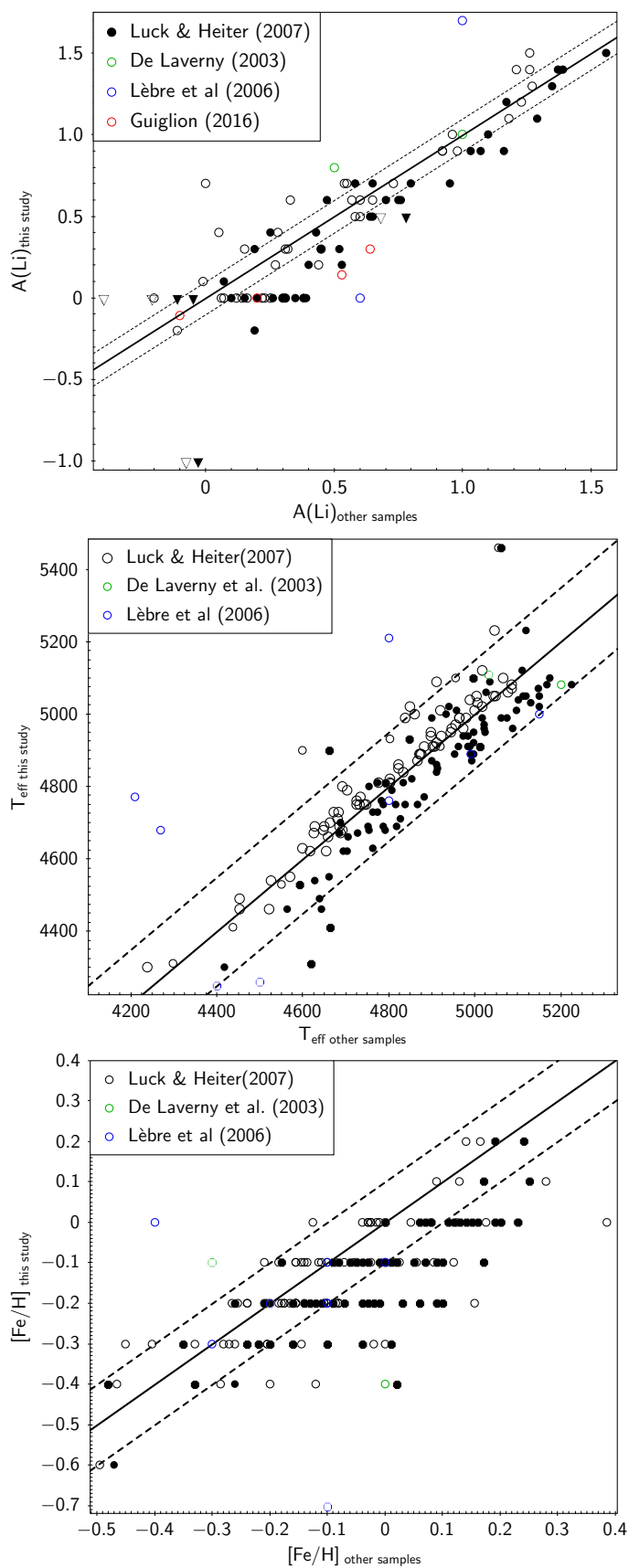

Fig. 6. Top: comparison between LTE Li abundances determined in this work and LTE Li abundances published in studies of de Laverny et al. (2003, green), Lèbre et al. (2006, blue), Luck \& Heiter (2007, black), and Guiglion et al. (2016, red). We exclude the stars with upper limits for Li published in the literature, but we include our upper limits in the case of real determinations by the other studies. Middle and bottom: comparison with effective temperature and $[\mathrm{Fe} / \mathrm{H}]$ values of same studies when available. For the sample of Luck \& Heiter (2007), we show their photometric and spectroscopic effective temperatures and corresponding $\mathrm{Li}$ and $[\mathrm{Fe} / \mathrm{H}]$ values (open and full black circles). Dotted lines represent a difference of $0.1 \mathrm{dex}$ in $\mathrm{Li}$ and $[\mathrm{Fe} / \mathrm{H}]$, and of $150 \mathrm{~K}$ in $T_{\text {eff }}$.

for the errors in effective temperature and luminosity given in Table 1 for our sample stars, as well as for the errors given in the original papers for the other samples; for all the samples we assume $[\mathrm{Fe} / \mathrm{H}]$ error of $0.1 \mathrm{dex}$. The values we derived for the mass and the corresponding uncertainty are given in Table 1 (see also Fig. 7) for our sample stars and in Table 2 for the three other samples. For the GALAH survey, we derived the stellar

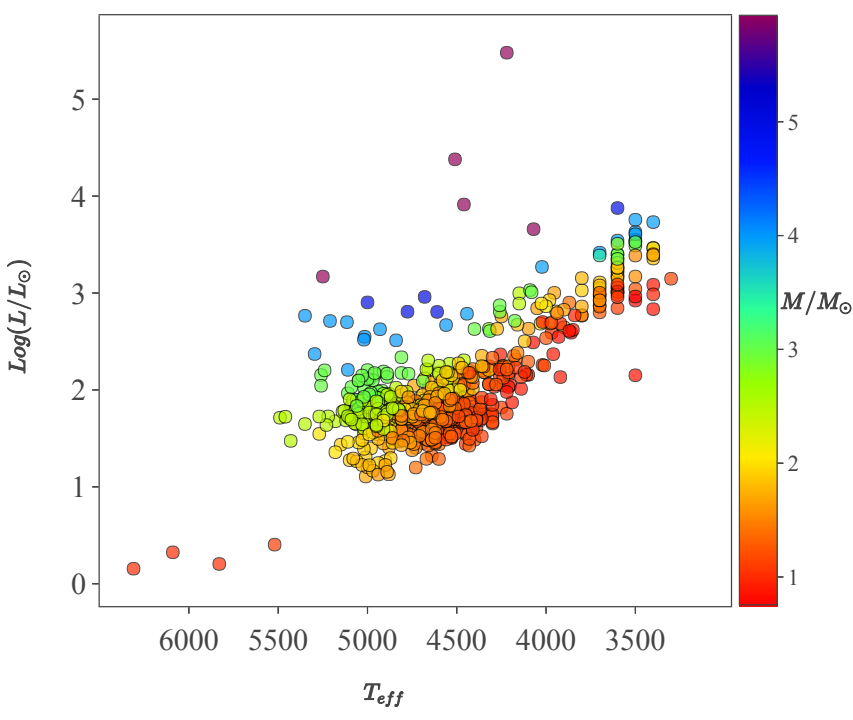

Fig. 7. Current masses of our sample stars as derived in Sect. 4.1.

mass for giant stars only (1734 stars). Because evolved stars lie in the region of the HRD where all the evolution tracks converge, and because of non-negligible error bars in effective temperature, luminosity, and metallicity, the masses we derive are relatively uncertain, as shown by the error bars we derive. None of the sample stars has an asteroseismic mass. Fortunately, very precise determination of the stellar mass of individual stars is not mandatory for this work, as we shall simply compare the $\mathrm{Li}$ abundance of stars in different domains of mass with the corresponding model predictions (Sect. 4.2).

Our sample covers the largest mass and advanced evolution ranges (compare Figs. 8-10). It contains stars with masses between $\sim 0.8$ and $6 \mathrm{M}_{\odot}$, and evolution stages from the end of the Hertzsprung gap to the early-AGB, the majority lying in the region of the RGB bump and of the clump, and on the upper part of the RGB, as a result of our selection criteria. On the other hand, the samples of Luck \& Heiter (2007) and Liu et al. (2014) focus on a more restricted mass range (between 1.5 and $4 \mathrm{M}_{\odot}$, and between 1.7 and $5 \mathrm{M}_{\odot}$ respectively; Fig. 9). Additionally, most of their stars appear to lie around the clump, although Luck \& Heiter (2007) also include LIMS (1.25 to $\left.2 \mathrm{M}_{\odot}\right)$ located between the MS turnoff and the RGB base. Finally, GALAH sample (Fig. 10) contains many MS stars, as well as low-mass evolved stars with luminosities much lower than that of the RGB tip.

\subsection{Li behaviour - The impact of rotation-induced mixing and thermohaline mixing}

We compare the Li behaviour of our sample stars and of the samples of Luck \& Heiter (2007), Liu et al. (2014), and GALAH (Buder et al. 2018) with the predictions from the grid of rotating stellar models of Lagarde et al. (2012). These models take into account thermohaline mixing as described by Charbonnel \& Zahn (2007) and Charbonnel \& Lagarde (2010), and rotation-induced processes following the formalism by Zahn (1992) and Maeder \& Zahn (1998). The initial rotation velocities assumed in the computations were chosen to lead to a good agreement with the observed range of rotation velocities in field and open cluster MS stars in the mass range of our sample (Gaige 1993; Zorec \& Royer 2012; see Charbonnel \& Lagarde 2010; Lagarde et al. 2012 for details). The corresponding 

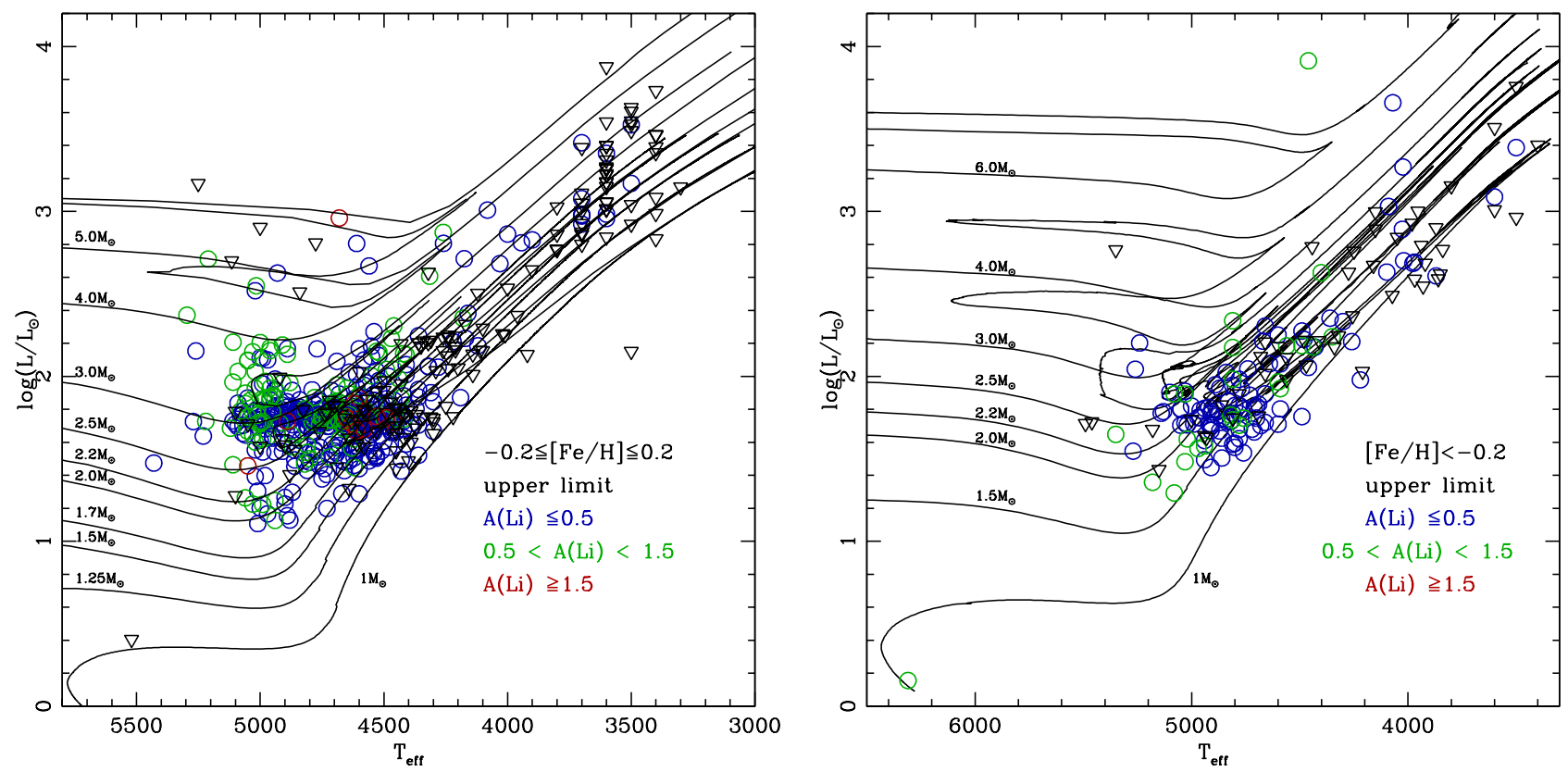

Fig. 8. Position of our sample stars in HRD with colour-labelled indication of Li (LTE) range as in Fig. 4. The sample is split in two metallicity ranges $([\mathrm{Fe} / \mathrm{H}]$ above and below $-0.2 \mathrm{dex}$, left and right respectively). The theoretical evolution tracks of the standard models (no rotation) of Lagarde et al. (2012) are superimposed for stars with masses between 1 and $6 \mathrm{M}_{\odot}$ computed with $[\mathrm{Fe} / \mathrm{H}]=0$ and -0.56 dex (left and right, respectively); we removed the two outliers with errors on the parallax larger than $70 \%$.
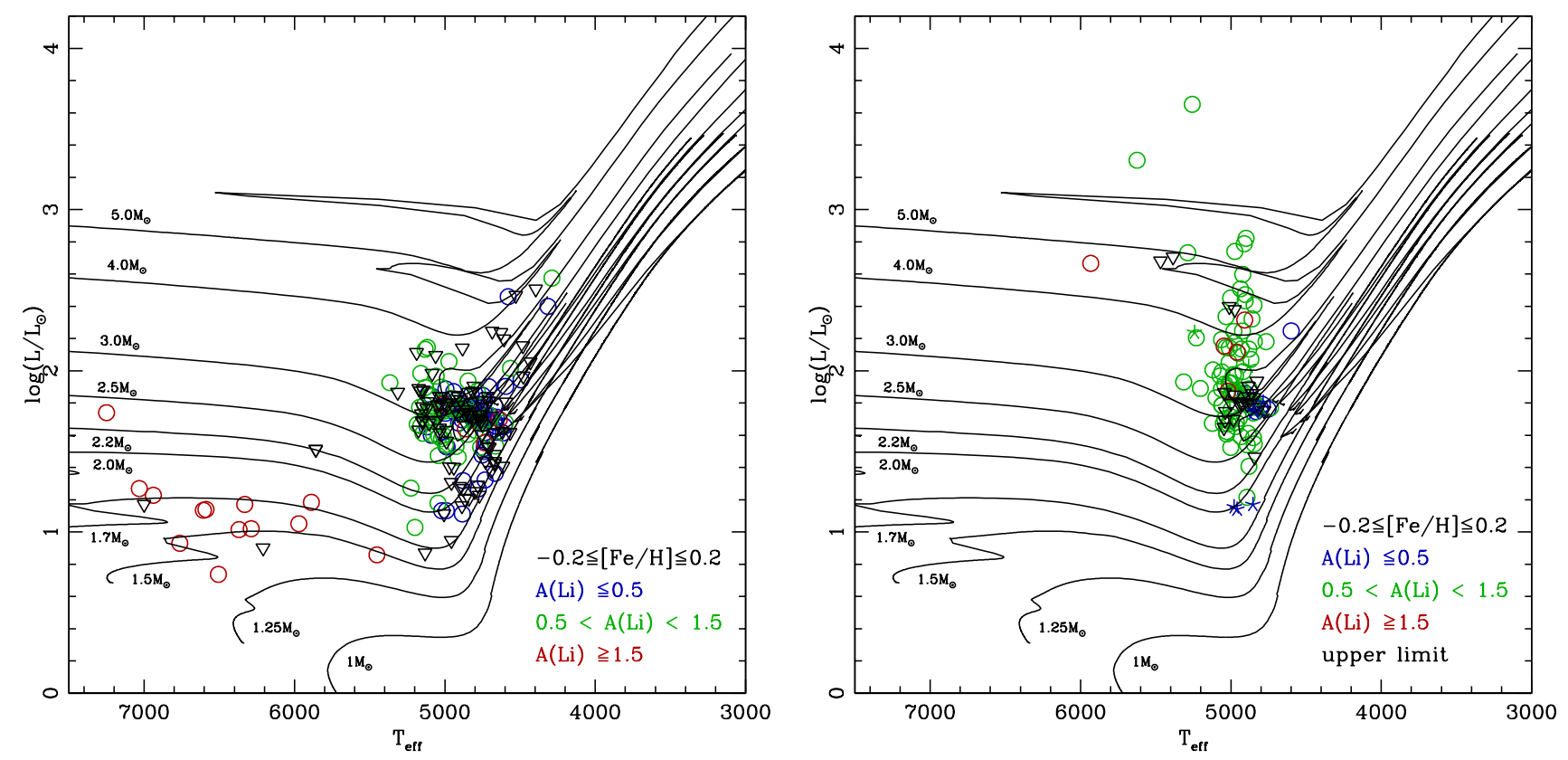

Fig. 9. Same as Fig. 8 but for samples observed by Luck \& Heiter (2007, LTE, left) and Liu et al. (2014, NLTE, right, with crosses indicating planet host stars). Here we show only their stars with $-0.2 \leq[\mathrm{Fe} / \mathrm{H}] \leq+0.2$ that have Gaia DR2 parallaxes (290 and 375 stars for Luck \& Heiter 2007; Liu et al. 2014, respectively). Evolution tracks are the standard models of Lagarde et al. (2012) computed with [Fe/H] $=0$.

evolution tracks are very similar to the standard tracks used in Sect. 4.1 to estimate the mass and evolution stage of the sample stars, hence no correction is needed with respect to these two quantities. However, thermohaline and rotation-induced mixing processes are necessary to account for the $\mathrm{Li}$ behaviour along the explored evolution sequence over the entire mass range considered. This is shown in Figs. 11-16, where the model predictions are compared to the $\mathrm{Li}$ observations, with effective temperature as a proxy for the evolution from the subgiant branch to the advanced phases (i.e., from high towards low temperatures).
Standard model predictions are also shown for discussion. In each figure panel, we select stars in a given mass and metallicity range, and compare them with the relevant theoretical tracks that are shown from the zero age main sequence up to the AGB phase.

\subsubsection{Our sample}

Let us focus first on our own sample, which we split in two metallicity ranges. In Fig. 11 we gather the Li data for our 

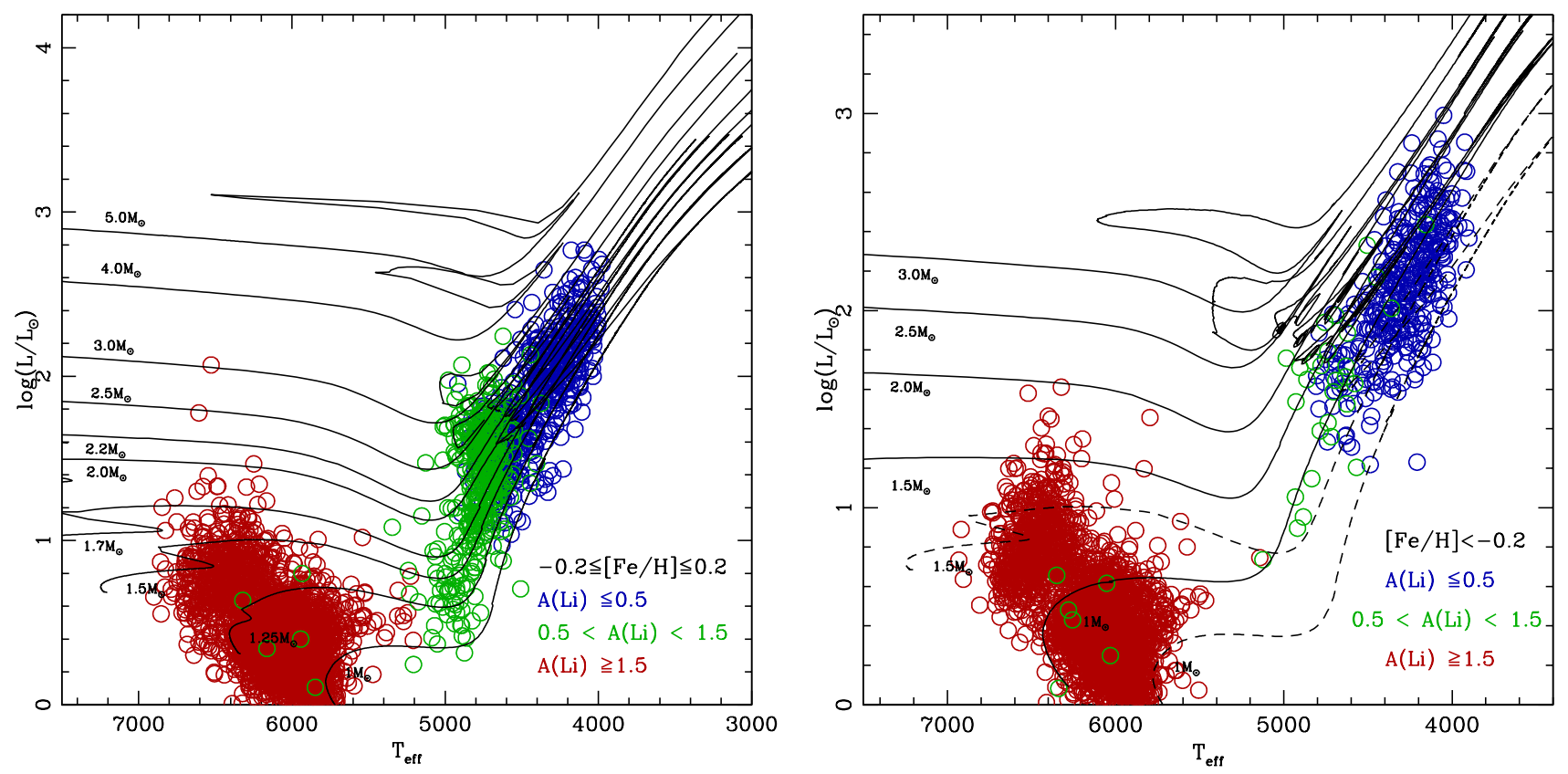

Fig. 10. Same as Fig. 8 for GALAH sub-samples with high accuracy or level of confidence in NLTE Li abundance (i.e. with Flag $_{\text {Cannon }}=0$ and Flag $\left.\mathrm{Li}_{\mathrm{Li}} \leq 1\right)$ and derived stellar parameters (Gaia DR2 parallaxes with errors less than $20 \%$, and errors on $T_{\text {eff }}$ less than $150 \mathrm{~K}$ ), and with $-0.2 \leq[\mathrm{Fe} / \mathrm{H}] \leq+0.2$ and $[\mathrm{Fe} / \mathrm{H}]<-0.2$ (left and right respectively). Evolution tracks are the standard models of Lagarde et al. (2012) computed with $[\mathrm{Fe} / \mathrm{H}]=0($ left $)$, and $[\mathrm{Fe} / \mathrm{H}]=0$ and -0.56 (right, dashed and solid respectively).

sample stars with $[\mathrm{Fe} / \mathrm{H}]$ between +0.2 and -0.2 dex (inclusive) and compare their behaviour with theoretical predictions for stellar models computed with $[\mathrm{Fe} / \mathrm{H}]=0$ dex. In Fig. 12 we show the Li data for the remaining stars with $[\mathrm{Fe} / \mathrm{H}]$ strictly lower than -0.2 dex and use stellar models computed with $[\mathrm{Fe} / \mathrm{H}]=-0.56 \mathrm{dex}$. Note that for these two values of $[\mathrm{Fe} / \mathrm{H}]$ the initial $\mathrm{Li}$ abundance assumed in the models is 3.11 and $2.49 \mathrm{dex}$ respectively, to account for Galactic chemical evolution (e.g. Romano et al. 1999; Ryan et al. 2001; Lambert \& Reddy 2004; Guiglion et al. 2016; Fu et al. 2018). In both cases we separate the stars with masses $\leq$ and $>2 \mathrm{M}_{\odot}$ and compare them with models of relevant initial stellar masses.

We first note that the Li surface evolution for the standard (non-rotating, i.e. $V_{\text {ZAMS }}=0$ ) models is very similar for stars of different masses and metallicities. In this case, surface Li depletion is due solely to the first dredge-up, and it starts relatively late in the Hertzsprung gap, that is, for $T_{\text {eff }}$ between $\sim 5200$ and $5400 \mathrm{~K}$. The predicted decrease in $\mathrm{Li}$ due to the first dredge-up is of the order of a factor 30-60. This depletion factor depends only on the maximum depth in mass reached by the convective envelope at the end of the dredge-up. It is independent of the initial Li value assumed in the models and it hardly depends on the stellar mass and metallicity. As a consequence, the minimum $\mathrm{Li}$ value obtained at the end of the first dredge-up episode is of the order of $\mathrm{A}(\mathrm{Li}) \sim 1.3-1.6$ for the models with $[\mathrm{Fe} / \mathrm{H}]=0$ and $\mathrm{A}(\mathrm{Li}) \sim 0.7-1$ for the models with $[\mathrm{Fe} / \mathrm{H}]=-0.56$. These standard predictions are in very good agreement with literature models (e.g. Iben 1967; Palacios et al. 2006; Charbonnel \& Zahn 2007; Mucciarelli et al. 2012), but at odds with the data. Clearly, the surface Li depletion observed starts earlier (i.e., for higher $\left.T_{\text {eff }}\right)$ and is much more efficient than predicted by the standard models that account only for dilution during the first dredge-up. This confirms earlier findings (e.g. Alschuler 1975; Brown et al. 1989; Balachandran 1990; do Nascimento et al. 2000; Palacios et al. 2003).

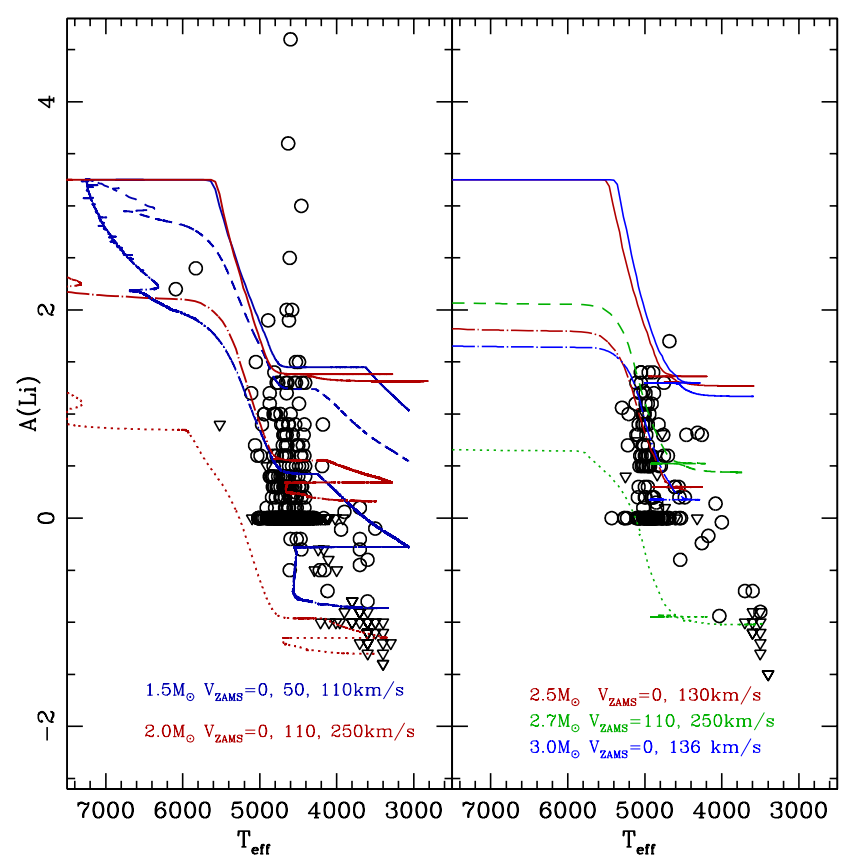

Fig. 11. Li data (LTE) for our solar-metallicity subsample $(-0.2 \leq[\mathrm{Fe} / \mathrm{H}] \leq+0.2 ;$ circles and triangles: $\mathrm{Li}$ determinations and upper limits) as a function of effective temperature as proxy for evolution (from high to cool values). Tracks show the predictions for $\mathrm{Li}$ behaviour as the stars evolve from the subgiant branch to the RGB tip and later from the clump to AGB phase from the non-rotating models with thermohaline mixing (solid) and the models including rotation and thermohaline mixing (others) from Lagarde et al. (2012) computed for $[\mathrm{Fe} / \mathrm{H}]=0$. At this metallicity, the initial Li abundance assumed in the models is 3.11 dex. In the left and right panels we plot respectively the stars with masses $\leq$ and $>2 \mathrm{M}_{\odot}$. The initial masses of the models are colour-labeled, with information on the initial (ZAMS) rotation velocity. 


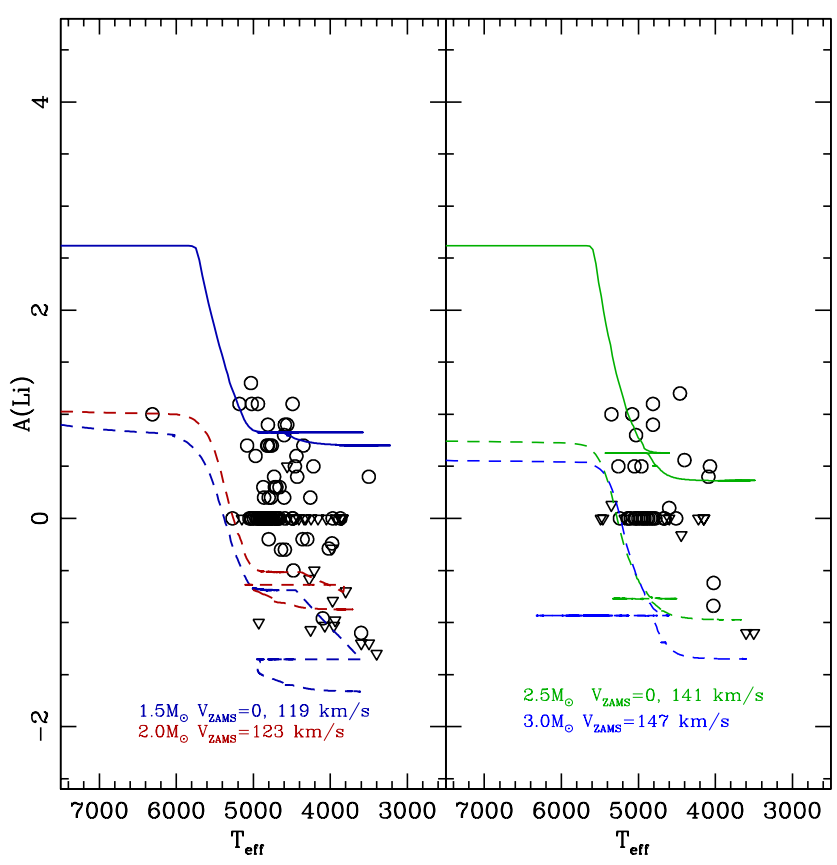

Fig. 12. Same as Fig. 11 for our low-metallicity sub-sample $([\mathrm{Fe} / \mathrm{H}]<-0.2)$ and non-rotating models with thermohaline mixing (solid) and models including rotation and thermohaline mixing (others) of Lagarde et al. (2012) computed for $[\mathrm{Fe} / \mathrm{H}]=-0.56$. At this metallicity, the initial $\mathrm{Li}$ abundance assumed in the models is 2.49 dex.

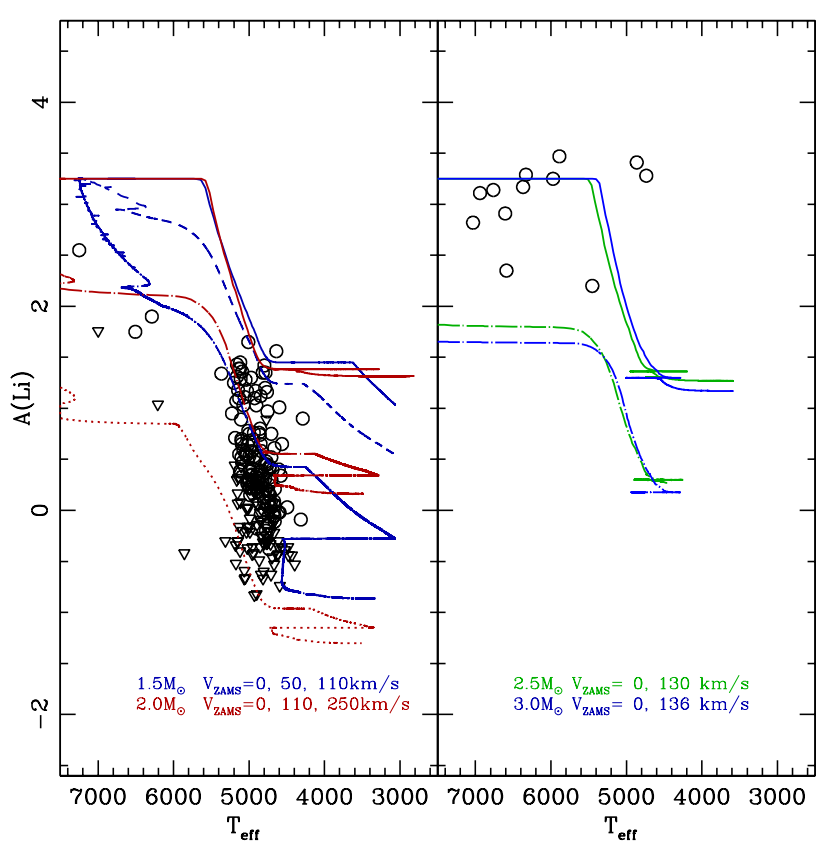

Fig. 13. Same as Fig. 11 (LTE abundances) for samples observed by Luck \& Heiter (2007). We show only their stars with $-0.2 \leq[\mathrm{Fe} / \mathrm{H}] \leq 0.2$ and compare with solar metallicity models including rotation and thermohaline mixing from Lagarde et al. (2012) computed for $[\mathrm{Fe} / \mathrm{H}]=0$.

On the other hand, the global behaviour and the dispersion of the $\mathrm{Li}$ abundances observed for the entire sample at all effective temperatures are very well reproduced by the rotating models. In this case, surface Li depletion starts much earlier (i.e., at a higher effective temperature at the entrance onto the Hertzsprung gap) than in the standard models. This is due to the enlargement of the

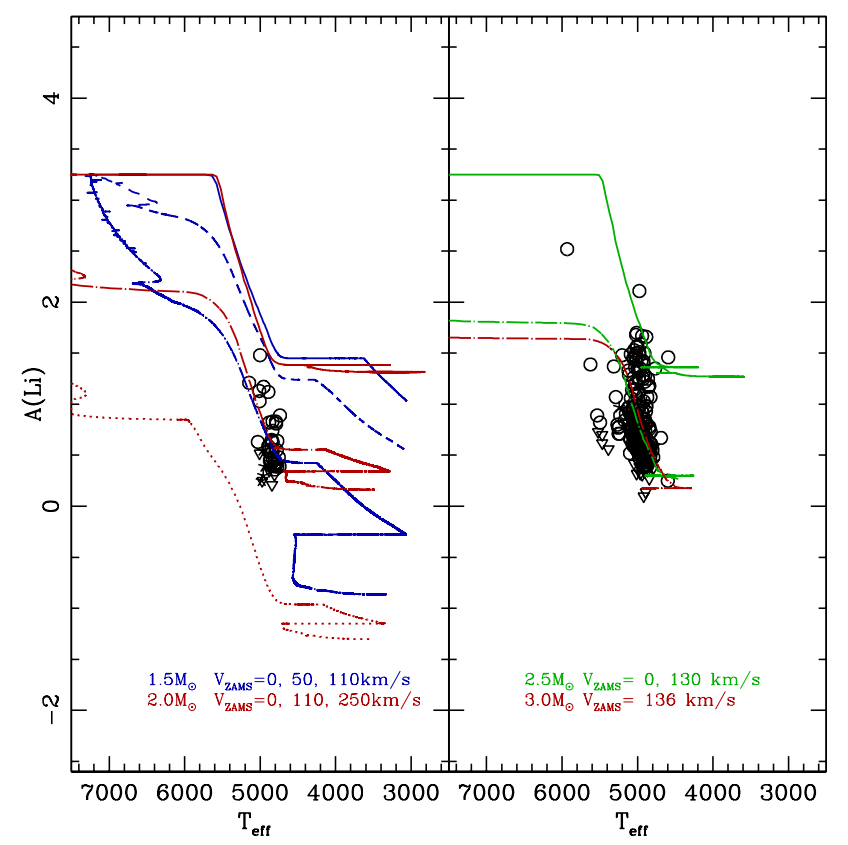

Fig. 14. Same as Fig. 11 for samples observed by Liu et al. (2014); in this case, Li data is NLTE. We show only their stars with $-0.2 \leq[\mathrm{Fe} / \mathrm{H}] \leq 0.2$ and compare with solar metallicity models including rotation and thermohaline mixing from Lagarde et al. (2012) computed for $[\mathrm{Fe} / \mathrm{H}]=0$.

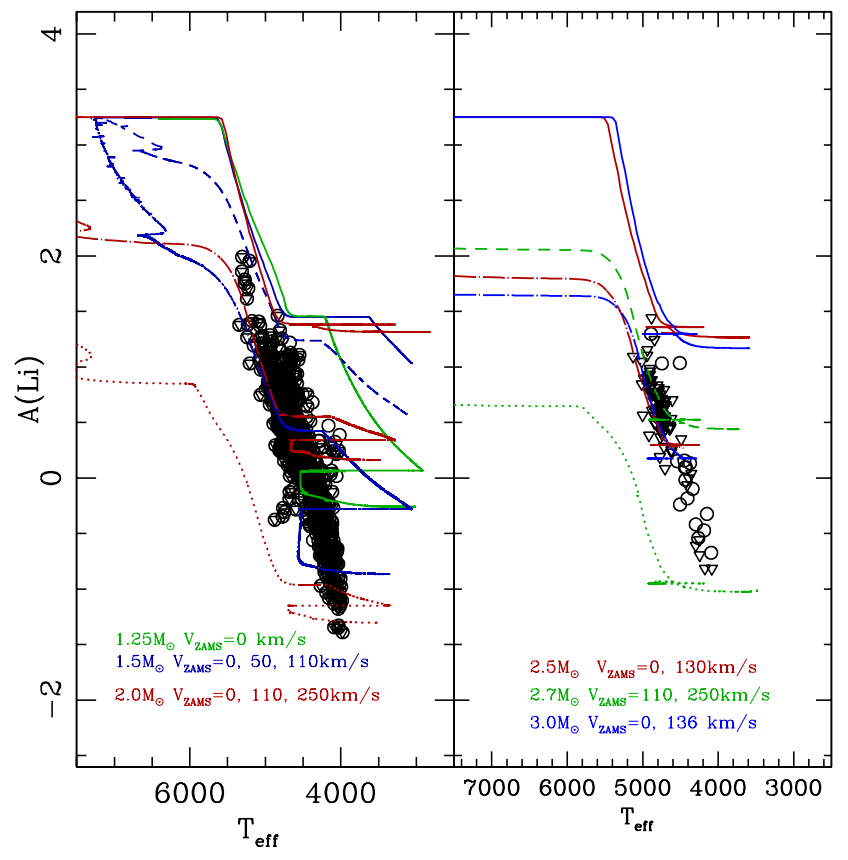

Fig. 15. Same as Fig. 11 for GALAH subsample of giant stars with $-0.2 \leq[\mathrm{Fe} / \mathrm{H}] \leq 0.2$ compared to predictions of models including rotation and thermohaline mixing computed with $[\mathrm{Fe} / \mathrm{H}]=0$. In this case, Li data is NLTE.

Li-free region by rotation-induced mixing while the stars are still on the MS. This also leads to more substantial Li depletion at the end of the first dredge-up event, as already anticipated with the first generation of rotating models of Palacios et al. (2003, also calculated with STAREVOL but with a less sophisticated treatment of rotation). For a given stellar mass, the higher the initial rotating rate, the lower the $\mathrm{Li}$ abundance all along the evolution from the MS turnoff to the RGB and the AGB. Clearly, 


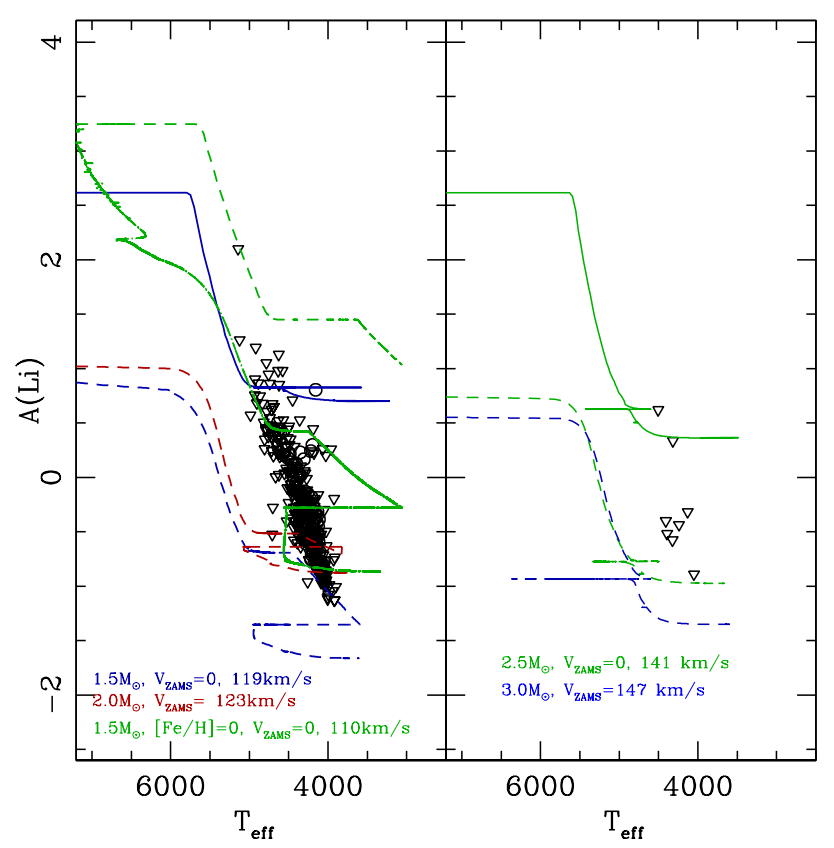

Fig. 16. Same as Fig. 15 for the GALAH sub-sample of giant stars with $[\mathrm{Fe} / \mathrm{H}]<-0.2$ compared to predictions of models including rotation and thermohaline mixing computed with $[\mathrm{Fe} / \mathrm{H}]=-0.5$ (and 0 green lines on the left panel only).

rotation-induced mixing brings the models in very good agreement with the Li data over the entire mass range, with the dispersion being explained by the assumed spread in initial rotation rate. As mentioned in Sect. 3.3, NLTE corrections would amount to only 0.1 to $0.3 \mathrm{dex}$, with the highest values for the cooler stars, that is, in the effective temperature regime where we already have lots of upper limits for Li. Hence, the above conclusions are not statistically affected by the lack of corrections (see also Sect. 4.2.4).

Additionally, for the low-mass range (below $\sim 2 \mathrm{M}_{\odot}$ ) thermohaline mixing occurs after the end of the first dredge-up event once the models reach the so-called RGB luminosity bump (which occurs at $T_{\text {eff }} \sim 4400-4300 \mathrm{~K}$, see Figs. 11 and 12, left panels). This leads to further Li depletion, as thermohaline mixing quickly transports $\mathrm{Li}$ from the convective envelope to hotter regions where it efficiently burns. The corresponding transport coefficient is obtained with the prescription for the thermohaline diffusion coefficient "à la Ulrich \& Kippenhahn” advocated by Charbonnel \& Zahn (2007). The excellent agreement between the Li data of low-mass stars that are more evolved than the RGB bump and our models provides strong support to our prescription for thermohaline mixing, despite existing tension with numerical simulations of this process (e.g. Denissenkov 2010b; Traxler et al. 2011; Sengupta \& Garaud 2018). The same coefficient also accounts for the observed behaviour of the carbon isotopic ratio and of the abundances of $\mathrm{C}$ and $\mathrm{N}$ in the upper part of the RGB in Population I and II stars (Charbonnel \& Zahn 2007; Charbonnel \& Lagarde 2010; Lagarde et al. 2012), and for the $\mathrm{C}$ and $\mathrm{N}$ abundances of giant stars investigated in the GaiaESO survey (Lagarde et al. 2019). It also leads to a significant reduction of the production of ${ }^{3} \mathrm{He}$ in low-mass stars, in better agreement with the behaviour of the evolution of this light element in the Galaxy (Charbonnel \& Zahn 2007; Lagarde et al. 2011).

In more massive stars (above $\sim 2 \mathrm{M}_{\odot}$; Figs. 11 and 12, right panels), thermohaline mixing is not expected to occur, because the stars do not pass through the RGB bump as they ignite central He burning earlier in non degenerate conditions. For the upper mass range, the lowest $\mathrm{Li}$ abundance observed can simply be explained by higher initial rotation rates, as already discussed above. Again, the above conclusions for the upper part of the RGB (both for the low-mass and the more massive stars) would not be affected by NLTE corrections, since most of the cooler stars have only Li upper limits.

\subsubsection{Comparison with Luck \& Heiter (2007) and Liu et al. (2014) samples}

For the samples of Luck \& Heiter (2007) and Liu et al. (2014) we considered only the stars that have both parallaxes and $V$ magnitudes from Gaia DR2 (290 out of 297 stars for Luck \& Heiter 2007 and 375 out of 378 for Liu et al. 2014). In this case, we used bolometric corrections from Alonso et al. (1999) to compute the luminosities. The mass (and the error on the mass) of the sample stars were determined as described in Sect. 4.1 and are listed in Table 2.

The predictions of the same rotating models at solar metallicity are compared in Figs. 13 and 14 to the observations of the stars with $[\mathrm{Fe} / \mathrm{H}]$ between +0.2 and -0.2 (inclusive) of the samples of Luck \& Heiter (2007) and Liu et al. (2014). In the first case, the $\mathrm{Li}$ abundances are LTE, while in the second case we show the NLTE abundances provided by the authors. We find similarly good agreement for both, better than in Sect. 4.2.1. Although we unfortunately have no stars in common with Liu et al. (2014), we see that the inclusion of NLTE corrections does not affect our global conclusions. In addition to the conclusions derived with our own sample, the Li data of the less evolved stars of Luck \& Heiter (2007) confirm the importance of rotation-induced mixing for the evolution of the surface Li abundance already at the MS turnoff and along the Hertzsprung gap.

\subsubsection{Comparison to GALAH sample with Gaia DR2 parallaxes}

In DR2 of the GALAH survey (Galactic Archaeology with HERMES; Buder et al. 2018), NLTE Li abundances were delivered for 342682 stars, most of them having Gaia DR2 parallaxes. For comparison with stellar evolution models predictions, we considered only GALAH stars with high accuracy or level of confidence in derived stellar parameters and $\mathrm{Li}$ abundance (i.e., with Flag $\operatorname{Cannon}=0$ and Flag $\mathrm{Li}_{\mathrm{Li}} \leq 1$ ), Gaia DR2 parallaxes with errors less than $20 \%$, and errors on $T_{\text {eff }}$ less than $150 \mathrm{~K}$. These selection criteria yielded a total of 7829 stars (MS and giants). We used bolometric corrections from Alonso et al. (1999) to compute the luminosities. The mass (and the error on the mass) of the sample stars was determined as described in Sect. 4.1. The positions of the stars in the HRD are compared with the standard evolution tracks of Lagarde et al. (2012) in Fig. 10 (we show only the stars with $[\mathrm{Fe} / \mathrm{H}] \leq+0.2$ ).

For the comparison with model predictions for $\mathrm{Li}$, we

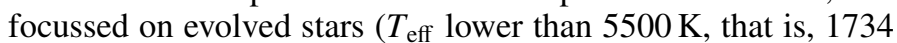
stars in total). To test for metallicity effects, we select two subsamples with $[\mathrm{Fe} / \mathrm{H}]$ between -0.2 and +0.2 (inclusive; 996 stars) on the one hand and $[\mathrm{Fe} / \mathrm{H}]$ below -0.2 (391 stars) on the other hand. The behaviour of the NLTE Li abundance along evolution is shown in Figs. 15 and 16, with relevant models for different mass ranges.

A striking relation becomes apparent between $\mathrm{A}(\mathrm{Li})$ and $T_{\text {eff }}$, which is not observed in any of the other samples. Thus, we looked for the differences in the data that could explain this 


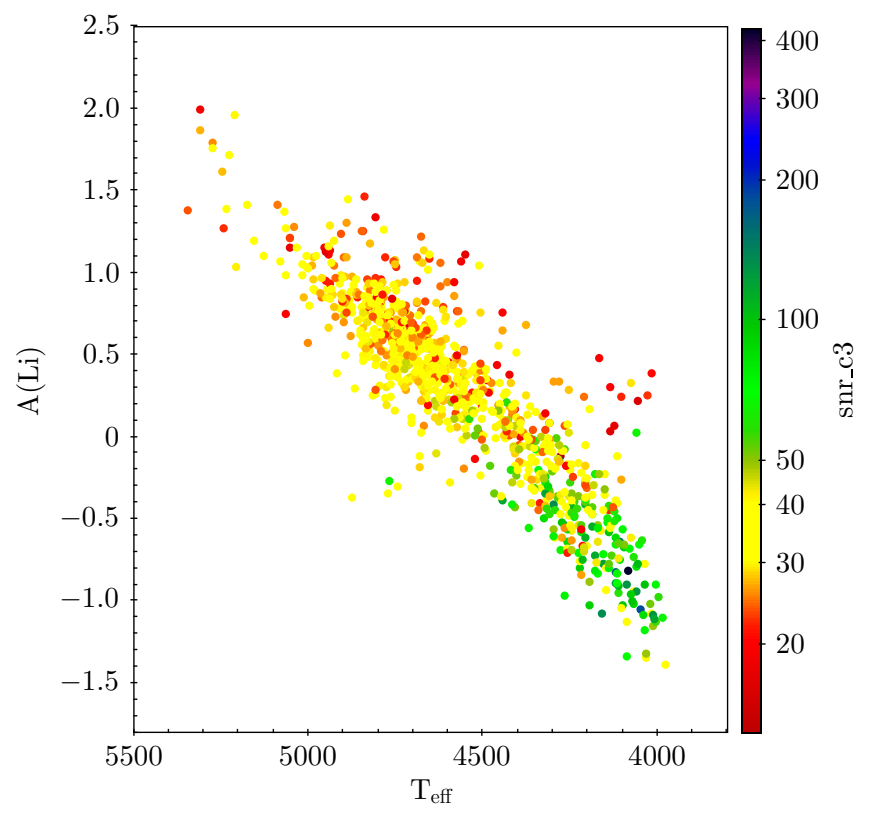

Fig. 17. Colour-coded $\mathrm{S} / \mathrm{N}$ from Buder et al. (2018) for the GALAH sub-sample selected with the criteria described in the text.

feature. We recall that our sample and the GALAH sample have no star in common, and that their spectral resolution $(R \sim 28000)$ is lower than ours (see Sect. 3.1). We checked the $\mathrm{S} / \mathrm{N}$ given by Buder et al. (2018) and, in particular, their snr_c3 which corresponds to the HERMES red channel covering the relevant wavelength range for $\mathrm{Li}$. As can be seen in Fig. 17, S/N is of the order of 20-30 for the large majority of the GALAH sub-sample stars. Only nine stars have high $S / N \sim 150$ as in our own data, out of which only four have a metallicity close to solar; these are also the stars with the lowest derived $\mathrm{Li}$ abundance and with the lowest effective temperature. If we keep the GALAH stars with the highest $\mathrm{S} / \mathrm{N}$, the sample obviously becomes too small to carry any statistical significance. On the other hand, the $\mathrm{A}(\mathrm{Li})$ and $T_{\text {eff }}$ relation relies on very low $\mathrm{S} / \mathrm{N}$ data, with the few high $\mathrm{S} / \mathrm{N}$ stars having very low Li. This apparent relation is thus highly questionable in the context of the criteria that form the basis our own work, in particular, the analysis of high $\mathrm{S} / \mathrm{N}$, high resolution spectra.

\subsubsection{LTE versus NLTE}

Correcting the $\mathrm{Li}$ abundances for NLTE effects would raise the representative points in Figs. $11-13$ by about 0.1 to 0.33 dex along the $y$-axis. This would not change the above conclusions obtained using LTE Li abundances, especially as they are based on a whole distribution of observed $\mathrm{Li}$ abundances, many of which are upper limits. A comparison between Figs. 11-16 demonstrates that our conclusions di indeed remain valid for studies providing NLTE Li abundances as well as for those providing LTE Li abundances. Thus, NLTE corrections do not appear crucial in our context; this could be expected since the NLTE corrections amount to a few tenths of a dex, while the effects of mixing reach about 2 dex.

\subsection{Li-rich stars}

\subsubsection{Li-rich giants: where do we stand?}

Li-rich giants (LiRG) are usually defined as evolved stars with $\mathrm{A}(\mathrm{Li})$ higher than $1.5 \mathrm{dex}$, which is the mean post dredge-up value predicted by standard evolution for Population I stars (see
Sect. 4.2.1). They are distinct from AGB stars producing Li through the Cameron \& Fowler (1971) mechanism during the thermal pulse phase (e.g. Smith \& Lambert 1989; Forestini \& Charbonnel 1997; Domínguez et al. 2004). Since the discovery of the first LiRG (Wallerstein \& Sneden 1982), several surveys have reported the existence of such objects which account for $\sim 1-2 \%$ of the studied populations (Brown et al. 1989; Charbonnel \& Balachandran 2000; Reddy \& Lambert 2005; Lèbre et al. 2006; Gonzalez et al. 2009; Kumar et al. 2011; Monaco et al. 2011; Lyubimkov et al. 2012; Casey et al. 2016; Smiljanic et al. 2018; Yan et al. 2018; Singh et al. 2019; Deepak 2019).

Some studies use HIPPARCos or Gaia data to determine the evolutionary status of field LiRG and show that these objects tend to accumulate close to the RGB bump, the clump, and the early-AGB (e.g. Charbonnel \& Balachandran 2000; Kumar et al. 2011; Smiljanic et al. 2018; Deepak 2019), which is in agreement with open cluster studies (e.g. Delgado Mena et al. 2016). Other works report, however, that LiRG can be randomly located in the HRD (Jasniewicz et al. 1999; Smith et al. 1999; Monaco et al. 2011; Lebzelter et al. 2012; Martell \& Shetrone 2013; Casey et al. 2016). The distinction is crucial to understanding the processes that may provide an explanation for the phenomenon, such as fresh $\mathrm{Li}$ production by internal mixing processes (Sackmann \& Boothroyd 1999; Palacios et al. 2001; Guandalini et al. 2009; Strassmeier et al. 2015; Cassisi et al. 2016), prompt mass loss events (de La Reza et al. 1996, 1997), Li accretion during engulfment of planets or planetesimals (Alexander 1967; Siess \& Livio 1999; Carlberg et al. 2010; Aguilera-Gómez et al. 2016a,b; Delgado Mena et al. 2016), tidal interactions between binary stars (Casey et al. 2019), or a combination of these mechanisms (Denissenkov \& Weiss 2000; Denissenkov \& Herwig 2004). However, since the evolution tracks of evolved stars all converge to the same area of the CMD, the definitive determination of the actual evolution status of LiRG requires asteroseismology to probe their internal structure and disentangle RGB from clump stars. As of today, very few LiRG have been observed with CoRoT and Kepler. The majority seems to be located in the core-He burning clump (Silva Aguirre et al. 2014; Bharat Kumar et al. 2018; Casey et al. 2016; Smiljanic et al. 2018; Singh et al. 2019), with the others being at the RGB bump or higher on the first ascent giant branch (Jofré et al. 2015; Casey et al. 2019). In a recent study using LAMOST spectra to derive both the $\mathrm{Li}$ abundance and asteroseismic classification, Casey et al. (2019) showed that $\sim 80 \%$ of their large sample of low-mass LiRG (2330 objects) probably have helium burning cores. They find that LiRG are more frequent at higher metallicity.

\subsubsection{Li-rich giants in the samples considered in the present study}

As mentioned above, the lower $\mathrm{Li}$ abundance for a LiRG is usually taken equal to $1.5 \mathrm{dex}$. This refers to a standard first dredge-up dilution factor of $\sim 60$, for a star with an initial cosmic abundance of 3.3 dex. Although this is in principle relevant for Pop I giant stars that have already undergone the first dredge-up, this is not an appropriate criterion for less evolved stars that have not yet undergone full Li dilution. Additionally, more metal-poor stars were probably born with a lower initial $\mathrm{Li}$ abundance, with a value of $\sim 2.5$ dex for stars with $\sim 3$ times fewer metals than the Sun (discussion and references in Sect. 4.2.1). Finally, uncertainties in the actual Li abundances should be taken into account, including those related to NLTE corrections. 
Table 3. Information about LiRG from our sample that were identified as having a LTE Li abundance higher by +0.2 dex than prediction by standard models at the corresponding effective temperature.

\begin{tabular}{lccccccccc}
\hline \hline HD & $\begin{array}{c}T_{\text {eff }} \\
(\mathrm{K})\end{array}$ & $\begin{array}{c}e_{-} T_{\text {eff }} \\
(\mathrm{K})\end{array}$ & $\log \left(L / L_{\odot}\right)$ & $e_{-} L$ & $\begin{array}{c}{[\mathrm{Fe} / \mathrm{H}]} \\
(\mathrm{dex})\end{array}$ & $\begin{array}{c}\mathrm{A}(\mathrm{Li}) \\
(\mathrm{dex})\end{array}$ & $\begin{array}{c}\text { Mass } \\
\left(\mathrm{M}_{\odot}\right)\end{array}$ & $\begin{array}{c}e_{M[-1 \sigma]} \\
\left(\mathrm{M}_{\odot}\right)\end{array}$ & $\begin{array}{c}e_{M[+1 \sigma]} \\
\left(\mathrm{M}_{\odot}\right)\end{array}$ \\
\hline 3750 & 4610 & 110 & 1.67 & 0.02 & 0.0 & 2.5 & 1.49 & 0.5 & 1.0 \\
4042 & 5020 & 120 & 1.62 & 0.026 & -0.3 & 1.1 & 2.0 & 0.9 & 0.5 \\
30197 & 4490 & 110 & 1.75 & 0.04 & 0.2 & 1.5 & 1.68 & 0.62 & 1.3 \\
40827 & 4620 & 110 & 1.83 & 0.02 & -0.1 & 1.9 & 1.57 & 0.53 & 0.93 \\
71129 & 4460 & 90 & 3.91 & 0.07 & -0.6 & 1.2 & 5.92 & 0.0 & 0.03 \\
77361 & 4600 & 90 & 1.87 & 0.02 & 0.0 & 4.6 & 1.78 & 0.59 & 1.21 \\
83506 & 4810 & 110 & 2.34 & 0.03 & -0.5 & 0.9 & 2.98 & 0.69 & 1.02 \\
85563 & 4530 & 90 & 1.76 & 0.04 & 0.0 & 1.5 & 1.39 & 0.4 & 1.1 \\
86634 & 4650 & 100 & 1.74 & 0.02 & 0.0 & 2.0 & 1.63 & 0.64 & 1.03 \\
90507 & 5030 & 110 & 1.48 & 0.02 & -0.3 & 1.3 & 1.9 & 0.4 & 0.6 \\
90633 & 4580 & 90 & 1.68 & 0.02 & 0.0 & 2.0 & 1.42 & 0.43 & 1.07 \\
93859 & 4590 & 110 & 1.92 & 0.03 & -0.3 & 0.9 & 1.39 & 0.4 & 1.09 \\
106574 & 4490 & 90 & 2.19 & 0.03 & -0.4 & 1.1 & 1.53 & 0.55 & 0.96 \\
113049 & 4810 & 110 & 2.17 & 0.031 & -0.3 & 1.1 & 2.98 & 1.18 & 1.02 \\
115299 & 4630 & 110 & 1.71 & 0.02 & -0.2 & 3.6 & 1.29 & 0.34 & 0.6 \\
133086 & 4890 & 110 & 1.73 & 0.02 & -0.2 & 1.9 & 2.0 & 1.05 & 0.5 \\
138289 & 4460 & 100 & 1.72 & 0.02 & 0.2 & 3.0 & 1.39 & 0.41 & 1.1 \\
183912 & 4680 & 80 & 2.96 & 0.07 & -0.1 & 1.7 & 4.99 & 1.0 & 0.97 \\
188114 & 4810 & 250 & 1.98 & 0.04 & -0.4 & 0.9 & 1.87 & 0.75 & 1.11 \\
199437 & 4560 & 110 & 2.18 & 0.03 & -0.3 & 0.9 & 1.85 & 0.66 & 1.13 \\
206078 & 4940 & 80 & 1.58 & 0.02 & -0.6 & 1.1 & 1.49 & 0.61 & 0.4 \\
\hline
\end{tabular}

Table 4. Information about LiRG that were identified as having NLTE Li abundance higher by +0.2 dex than prediction by standard models at corresponding effective temperature in the sample of Luck \& Heiter (2007) and Liu et al. (2014).

\begin{tabular}{lcccccccc}
\hline \hline HD & $\begin{array}{c}T_{\text {eff }} \\
(\mathrm{K})\end{array}$ & $\log \left(L / L_{\odot}\right)$ & $e_{-} L$ & $\begin{array}{c}{[\mathrm{Fe} / \mathrm{H}]} \\
(\mathrm{dex})\end{array}$ & $\begin{array}{c}\mathrm{A}(\mathrm{Li}) \\
(\mathrm{dex})\end{array}$ & $\begin{array}{c}\text { Mass } \\
\left(\mathrm{M}_{\odot}\right)\end{array}$ & $\begin{array}{c}e_{M[-1 \sigma]} \\
\left(\mathrm{M}_{\odot}\right)\end{array}$ & $\begin{array}{c}e_{M[+1 \sigma]} \\
\left(\mathrm{M}_{\odot}\right)\end{array}$ \\
\hline 186815 & 5116 & 1.32 & 0.02 & -0.22 & 1.29 & 1.9 & 0.2 & 0.1 \\
214995 & 4737 & 1.55 & 0.02 & 0.0 & 3.01 & 1.59 & 0.75 & 0.9 \\
\hline \multicolumn{8}{c}{ Liu } & Let al. (2014) \\
\hline 5395 & 4774 & 1.75 & 0.03 & -0.45 & 1.3 & 1.39 & 0.54 & 0.5 \\
35410 & 4809 & 1.59 & 0.03 & -0.33 & 1.26 & 1.39 & 0.55 & 0.6 \\
102845 & 4975 & 2.01 & 0.03 & -0.07 & 2.11 & 2.99 & 0.5 & 0.01 \\
138905 & 4822 & 1.85 & 0.03 & -0.3 & 1.44 & 1.79 & 0.79 & 1.19 \\
160781 & 4593 & 2.6 & 0.04 & -0.02 & 1.46 & 3.99 & 1.01 & 1.0 \\
196857 & 4878 & 1.70 & 0.02 & -0.27 & 1.22 & 1.69 & 0.83 & 0.8 \\
\hline
\end{tabular}

It is for these reasons that we identify as LiRG those stars that have LTE Li abundance and 0.2 dex higher than the Li value predicted by the standard models at the corresponding effective temperature and for the relevant value of $[\mathrm{Fe} / \mathrm{H}]$ (see Fig. 11 and related figures). We chose this value of 0.2 dex as it corresponds to the typical uncertainty for the $\mathrm{Li}$ abundance. We provide information on the LiRGs of our own sample and that of Luck \& Heiter (2007) and Liu et al. (2014) in Tables 3 and 4 respectively. The position of all the LiRG we identified is shown in the HRD in Fig. 18.

In our own sample (Fig. 18 and Table 3) we identify seven LiRG that pass the "classical" criterion $(\mathrm{A}(\mathrm{Li}) \geq 1.5 \mathrm{dex})$. This corresponds to $0.9 \%$ of the stars we observed, which a typical percentage for LiRG found in the surveys of the literature. With our new criterion, we obtain twelve additional LiRG. The total nineteen stars correspond to $2.2 \%$ of our sample. Only two LiRG have Li higher than the cosmic value of $3.3 \mathrm{dex}$, one has a Li abun- dance of 3.0 dex; all the others have $\mathrm{A}(\mathrm{Li}) \leq 2.5 \mathrm{dex}$. All of them lie close to the RGB bump and the He-burning clump, except for two objects that are much brighter and more massive than the rest of the LiRG subsample, and which have relatively low $[\mathrm{Fe} / \mathrm{H}]$. The position of the low-mass LiRG is, thus, in agreement with the previous findings. Without asteroseismology however, no definitive conclusion can be obtained on their actual evolution state.

Upon applying our criterion, we find two and six LiRG in the samples of Liu et al. (2014) and Luck \& Heiter (2007), respectively. This corresponds to 0.5 and $2 \%$ of the total number of red giant stars considered from the respective surveys. We find no LiRG among our GALAH subsample while Deepak (2019) found $0.64 \% \mathrm{LiRG}$ and $0.03 \%$ super Li-rich giant $(\mathrm{A}(\mathrm{Li})>3.2)$ among a larger GALAH subsample selected using a less restrictive $\mathrm{Li}$ abundance flag than the one we adopted (Sect. 4.2.3). All the LiRG we identify in the literature samples lie close to the RGB bump or the He-burning clump except for one star that 


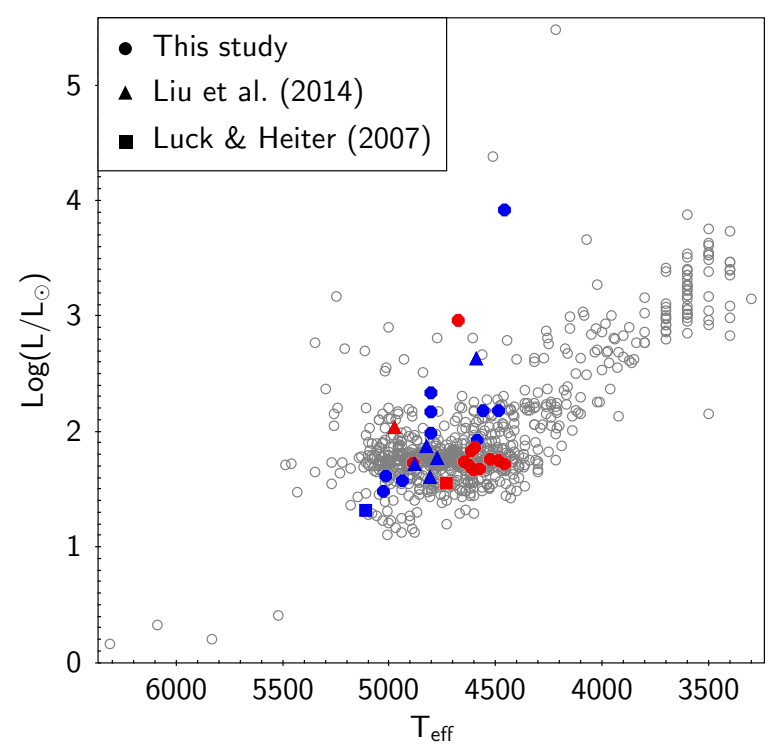

Fig. 18. Position in HRD of the LiRG identified with our adopted criterion. Red and blue stars are for $\mathrm{Li}$ values higher or lower than $1.5 \mathrm{dex}$, respectively.

is both brighter and more massive than the bulk LiRG sample. Again, no definitive conclusion can be obtained on their actual evolution state without asteroseismic constraints.

\subsubsection{In terms of planets}

The entire LiRG sample has been cross-matched with the current sample of 112 published substellar companions around giant stars, a list kept up-to-date by Sabine Reffert's Heidelberg team ${ }^{2}$ and no match was found. In order to avoid any error in the determination of the host evolutionary stage, we extended our search to the current sample of 4003 published confirmed exoplanets ${ }^{3}$, once again leading to no match.

\section{Conclusions}

$\mathrm{Li}$ is a fragile element that can be used as a tracer of transport processes in stellar interiors along the evolution of LIMS. Its photospheric abundance is known to vary extensively with initial stellar mass and evolution stage. Here we test stellar evolution models which predict stronger Li depletion for higher initial rotation in the advanced phases of the evolution as well as a strong impact of thermohaline mixing on low-mass, bright RGB stars. For this purpose, we derived Li abundances in a volume-limited sample of field giant stars with precise Gaia parallaxes and we also used literature samples with HIPPARCos or Gaia measurements. The model-data comparison confirms the impact of rotation-induced mixing and provides excellent support to our prescription for the efficiency of thermohaline mixing. Finally, we find only $0.8 \%$ of LiRG in our sample when using the classical definition used in the literature, and $2.2 \%$ when choosing a definition that accounts for the actual evolution stage of the stars with respect to the end of the first dredge-up. We can conclude that Gaia puts a new spin on how the mixing processes in stars are understood. However, asteroseismology is required to definitively pinpoint the actual evolution status of the LiRG.

\footnotetext{
2 https://exoplanetarchive.ipac.caltech.edu

3 http://www.1sw.uni-heidelberg.de/users/sreffert/ giantplanets/giantplanets.php
}

Acknowledgements. Part of this work was supported by the French "Programme National de Physique Stellaire" (PNPS) and "Programme National Cosmologie et Galaxies" (PNCG) of CNRS/INSU, and by the Swiss National Science Foundation (SNF). RS acknowledges support by the National Science Center of Poland through grant 2012/07/B/ST9/04428 and support from the Polish Ministry of Science and Higher Education. PN thanks Dr. J. Pritchard for his help with the FEROS pipeline. We thank R. Gauderon for having observed with FEROS@ESO in March 2004. JKH and observations taken at McDonald Observatory were supported by the McDonald REU program under NSF AST0649128. We thank the anonymous referee for important comments that helped improve the paper.

\section{References}

Aguilera-Gómez, C., Chanamé, J., Pinsonneault, M. H., \& Carlberg, J. K. 2016a, ApJ, 829, 127

Aguilera-Gómez, C., Chanamé, J., Pinsonneault, M. H., \& Carlberg, J. K. 2016b, ApJ, 833, L24

Alexander, J. B. 1967, The Observatory, 87, 238

Alonso, A., Arribas, S., \& Martínez-Roger, C. 1999, A\&AS, 140, 261

Alschuler, W. R. 1975, ApJ, 195, 649

Andrae, R., Fouesneau, M., Creevey, O., et al. 2018, A\&A, 616, A8

Anthony-Twarog, B. J., Deliyannis, C. P., Twarog, B. A., Croxall, K. V., \& Cummings, J. D. 2009, AJ, 138, 1171

Arenou, F., Luri, X., Babusiaux, C., et al. 2018, A\&A, 616, A17

Asplund, M., Grevesse, N., Sauval, A. J., \& Scott, P. 2009, ARA\&A, 47, 481

Balachandran, S. 1990, ApJ, 354, 310

Balachandran, S. 1995, ApJ, 446, 203

Bharat Kumar, Y., Singh, R., Eswar Reddy, B., \& Zhao, G. 2018, ApJ, 858, L22

Boesgaard, A. M. 1987, PASP, 99, 1067

Boesgaard, A. M., \& Tripicco, M. J. 1986, ApJ, 302, L49

Brown, J. A., Sneden, C., Lambert, D. L., \& Dutchover, Jr., E. 1989, ApJS, 71, 293

Buder, S., Asplund, M., Duong, L., et al. 2018, MNRAS, 478, 4513

Caffau, E., Ludwig, H. G., Steffen, M., \& Bonifacio, P. 2010, in Light Elements in the Universe, eds. C. Charbonnel, M. Tosi, F. Primas, \& C. Chiappini, IAU Symp., 268, 329

Cameron, A. G. W., \& Fowler, W. A. 1971, ApJ, 164, 111

Carlberg, J. K., Smith, V. V., Cunha, K., Majewski, S. R., \& Rood, R. T. 2010, ApJ, 723, L103

Carlos, M., Nissen, P. E., \& Meléndez, J. 2016, A\&A, 587, A100

Casey, A. R., Ruchti, G., Masseron, T., et al. 2016, MNRAS, 461, 3336

Casey, A. R., Ho, A. Y. Q., Ness, M., et al. 2019, ApJ, 880, 125

Cassisi, S., Salaris, M., \& Pietrinferni, A. 2016, A\&A, 585, A124

Castro, M., Do Nascimento, Jr., J. D., Biazzo, K., Meléndez, J., \& de Medeiros, J. R. 2011, A\&A, 526, A17

Charbonnel, C., \& Balachandran, S. C. 2000, A\&A, 359, 563

Charbonnel, C., \& Lagarde, N. 2010, A\&A, 522, A10

Charbonnel, C., \& Talon, S. 2005, Science, 309, 2189

Charbonnel, C., \& Zahn, J.-P. 2007, A\&A, 467, L15

Chen, Y. Q., Nissen, P. E., Benoni, T., \& Zhao, G. 2001, A\&A, 371, 943

Cui, X.-Q., Zhao, Y.-H., Chu, Y.-Q., et al. 2012, Res. Astron. Astrophys., 12, 1197

Cummings, J. D., Deliyannis, C. P., Maderak, R. M., \& Steinhauer, A. 2017, AJ, 153,128

de La Reza, R., Drake, N. A., \& da Silva, L. 1996, ApJ, 456, L115

de la Reza, R., Drake, N. A., da Silva, L., Torres, C. A. O., \& Martin, E. L. 1997, ApJ, 482, L77

de Laverny, P., do Nascimento, Jr., J. D., Lèbre, A., \& De Medeiros, J. R. 2003, A\&A, 410, 937

Deepak, R. B. E. 2019, MNRAS, 484, 2000

Delgado Mena, E., Israelian, G., González Hernández, J. I., et al. 2014, A\&A, 562, A92

Delgado Mena, E., Bertrán de Lis, S., Adibekyan, V. Z., et al. 2015, A\&A, 576, A69

Delgado Mena, E., Tsantaki, M., Sousa, S. G., et al. 2016, A\&A, 587, A66

Deliyannis, C. P., Pinsonneault, M. H., \& Charbonnel, C. 2000, in The Light Elements and their Evolution, eds. L. da Silva, R. de Medeiros, \& M. Spite, IAU Symp., 198, 61

Denissenkov, P. A. 2010a, ApJ, 719, 28

Denissenkov, P. A. 2010b, ApJ, 723, 563

Denissenkov, P. A., \& Herwig, F. 2004, ApJ, 612, 1081

Denissenkov, P. A., \& Weiss, A. 2000, A\&A, 358, L49

Denissenkov, P. A., Pinsonneault, M., \& MacGregor, K. B. 2009, ApJ, 696, 1823

Domínguez, I., Abia, C., Straniero, O., Cristallo, S., \& Pavlenko, Y. V. 2004, A\&A, 422, 1045 
do Nascimento, Jr., J. D., Charbonnel, C., Lèbre, A., de Laverny, P., \& De Medeiros, J. R. 2000, A\&A, 357, 931

Do Nascimento, Jr., J. D., Castro, M., Meléndez, J., et al. 2009, A\&A, 501, 687 Eggenberger, P., Haemmerlé, L., Meynet, G., \& Maeder, A. 2012, A\&A, 539, A70

Evans, D. W., Riello, M., De Angeli, F., et al. 2018, A\&A, 616, A4

Flower, P. J. 1996, ApJ, 469, 355

Forestini, M., \& Charbonnel, C. 1997, A\&AS, 123, 241

Fu, X., Romano, D., Bragaglia, A., et al. 2018, A\&A, 610, A38

Gaia Collaboration (Babusiaux, C., et al.) 2018a, A\&A, 616, A10

Gaia Collaboration (Brown, A. G. A., et al.) 2018b, A\&A, 616, A1

Gaige, Y. 1993, A\&A, 269, 267

Gillet, D., Burnage, R., Kohler, D., et al. 1994, A\&AS, 108, 181

Gonzalez, O. A., Zoccali, M., Monaco, L., et al. 2009, A\&A, 508, 289

Gratton, R. G., Sneden, C., Carretta, E., \& Bragaglia, A. 2000, A\&A, 354, 169

Guandalini, R., Palmerini, S., Busso, M., \& Uttenthaler, S. 2009, PASA, 26, 168

Guiglion, G., de Laverny, P., Recio-Blanco, A., et al. 2016, A\&A, 595, A18

Gustafsson, B., Edvardsson, B., Eriksson, K., et al. 2008, A\&A, 486, 951

Henkel, K., Karakas, A. I., \& Lattanzio, J. C. 2017, MNRAS, 469, 4600

Houdashelt, M. L., Bell, R. A., \& Sweigart, A. V. 2000, AJ, 119, 1448

Iben, Jr., I. 1967, ApJ, 147, 624

Jasniewicz, G., Parthasarathy, M., de Laverny, P., \& Thévenin, F. 1999, A\&A, 342,831

Jofré, E., Petrucci, R., García, L., \& Gómez, M. 2015, A\&A, 584, L3

King, J. R., Deliyannis, C. P., Hiltgen, D. D., et al. 1997, AJ, 113, 1871

Kumar, Y. B., Reddy, B. E., \& Lambert, D. L. 2011, ApJ, 730, L12

Lagarde, N., Charbonnel, C., Decressin, T., \& Hagelberg, J. 2011, A\&A, 536, A28

Lagarde, N., Decressin, T., Charbonnel, C., et al. 2012, A\&A, 543, A108

Lagarde, N., Robin, A. C., Reylé, C., \& Nasello, G. 2017, A\&A, 601, A27

Lagarde, N., Reylé, C., Robin, A. C., et al. 2019, A\&A, 621, A24

Lambert, D. L., \& Reddy, B. E. 2004, MNRAS, 349, 757

Lèbre, A., de Laverny, P., de Medeiros, J. R., Charbonnel, C., \& da Silva, L. 1999, A\&A, 345, 936

Lèbre, A., de Laverny, P., Do Nascimento, Jr., J. D., \& de Medeiros, J. R. 2006 , A\&A, 450, 1173

Lebzelter, T., Uttenthaler, S., Busso, M., Schultheis, M., \& Aringer, B. 2012, A\&A, 538, A36

Lind, K., Primas, F., Charbonnel, C., Grundahl, F., \& Asplund, M. 2009a, A\&A, 503,545

Lind, K., Asplund, M., \& Barklem, P. S. 2009b, A\&A, 503, 541

Lindegren, L., Hernández, J., Bombrun, A., et al. 2018, A\&A, 616, A2

Liu, Y. J., Tan, K. F., Wang, L., et al. 2014, ApJ, 785, 94

Lodders, K., Palme, H., \& Gail, H. P. 2009, Solar System, Landolt-Börnstein Group VI Astronomy and Astrophysics, 4B, 712

Luck, R. E., \& Heiter, U. 2007, AJ, 133, 2464
Lyubimkov, L. S., Lambert, D. L., Kaminsky, B. M., et al. 2012, MNRAS, 427, 11

Maeder, A., \& Zahn, J.-P. 1998, A\&A, 334, 1000

Mallik, S. V., Parthasarathy, M., \& Pati, A. K. 2003, A\&A, 409, 251

Martell, S. L., \& Shetrone, M. D. 2013, MNRAS, 430, 611

Martín, E. L., Basri, G., Pavlenko, Y., \& Lyubchik, Y. 2002, ApJ, 579, 437

Michaud, G. 1986, ApJ, 302, 650

Mikolaitis, Š., de Laverny, P., Recio-Blanco, A., et al. 2017, A\&A, 600, A22

Monaco, L., Villanova, S., Moni Bidin, C., et al. 2011, A\&A, 529, A90

Monroe, T. R., Meléndez, J., Ramírez, I., et al. 2013, ApJ, 774, L32

Mucciarelli, A., Salaris, M., \& Bonifacio, P. 2012, MNRAS, 419, 2195

Palacios, A., Charbonnel, C., \& Forestini, M. 2001, A\&A, 375, L9

Palacios, A., Talon, S., Charbonnel, C., \& Forestini, M. 2003, A\&A, 399, 603

Palacios, A., Charbonnel, C., Talon, S., \& Siess, L. 2006, A\&A, 453, 261

Pasquini, L., Randich, S., Zoccali, M., et al. 2004, A\&A, 424, 951

Perryman, M. A. C., Lindegren, L., Kovalevsky, J., et al. 1997, A\&A, 323, L49

Ramírez, I., Fish, J. R., Lambert, D. L., \& Allende Prieto, C. 2012, ApJ, 756, 46 Reddy, B. E., \& Lambert, D. L. 2005, AJ, 129, 2831

Richer, J., \& Michaud, G. 1993, ApJ, 416, 312

Romano, D., Matteucci, F., Molaro, P., \& Bonifacio, P. 1999, A\&A, 352, 117

Ryan, S. G., Kajino, T., Beers, T. C., et al. 2001, ApJ, 549, 55

Sackmann, I.-J., \& Boothroyd, A. I. 1999, ApJ, 510, 217

Schramm, D. N., Steigman, G., \& Dearborn, D. S. P. 1990, ApJ, 359, L55

Sengupta, S., \& Garaud, P. 2018, ApJ, 862, 136

Siess, L., \& Livio, M. 1999, MNRAS, 308, 1133

Silva Aguirre, V., Ruchti, G. R., Hekker, S., et al. 2014, ApJ, 784, L16

Singh, R., Reddy, B. E., Bharat Kumar, Y., \& Antia, H. M. 2019, ApJ, 878, L21

Smiljanic, R., Franciosini, E., Bragaglia, A., et al. 2018, A\&A, 617, A4

Smith, V. V., \& Lambert, D. L. 1989, ApJ, 345, L75

Smith, V. V., Shetrone, M. D., \& Keane, M. J. 1999, ApJ, 516, L73

Strassmeier, K. G., Carroll, T. A., Weber, M., \& Granzer, T. 2015, A\&A, 574, A31

Talon, S., \& Charbonnel, C. 2003, A\&A, 405, 1025

Talon, S., \& Charbonnel, C. 2005, A\&A, 440, 981

Talon, S., \& Charbonnel, C. 2010, in Light Elements in the Universe, eds. C. Charbonnel, M. Tosi, F. Primas, \& C. Chiappini, IAU Symp., 268, 365

Traxler, A., Garaud, P., \& Stellmach, S. 2011, ApJ, 728, L29

Valle, G., Dell'Omodarme, M., Prada Moroni, P. G., \& Degl'Innocenti, S. 2014, A\&A, 561, A125

van Leeuwen, F. 2007, A\&A, 474, 653

Wallerstein, G., \& Sneden, C. 1982, ApJ, 255, 577

Wallerstein, G., Herbig, G. H., \& Conti, P. S. 1965, ApJ, 141, 610

Yan, H.-L., Shi, J.-R., Zhou, Y.-T., et al. 2018, Nat. Astron., 2, 790

Zahn, J.-P. 1992, A\&A, 265, 115

Zorec, J., \& Royer, F. 2012, A\&A, 537, A120 